\title{
Advances in computational design and optimization with application to MEMS
}

\author{
Bing-Chung Chen ${ }^{1, * \dagger}$, Emílio C. N. Silva ${ }^{2}$ and Noboru Kikuchi ${ }^{1}$ \\ ${ }^{1}$ Department of Mechanical Engineering and Applied Mechanics, The University of Michigan, \\ Ann Arbor, MI 48109-2125, U.S.A. \\ ${ }^{2}$ Department of Mechanical Engineering, Escola Politécnica, Universidade de São Paulo, \\ São Paulo, SP 05508-900, Brazil
}

\begin{abstract}
SUMMARY
In this paper, we will highlight the current research which employs the topology optimization to find the optimal configuration of various smart structures and microstructures, specifically, pressure actuated compliant mechanisms, flextensional transducers, and porous material microstructures with unusual thermoelastic properties. These examples demonstrate that the topology optimization problem involving multiple physics domain is a viable direction for future research, in particular, for sensor and actuator design. Copyright (c) 2001 John Wiley \& Sons, Ltd.
\end{abstract}

KEY WORDS: topology optimization; compliant mechanism; pressure; flextensional actuators

\section{INTRODUCTION}

In the last decade, the field of structural topology optimization has expanded significantly, successfully addressing many practical engineering problems. As a result, this methodology has been widely accepted in industry, with several commercial software packages available $[1,2]$.

The optimal design of the topology of continuous structures is achieved by methods adopting the approach of material distribution over a fixed reference region. The underlying spirit is to represent the geometry of a structure by a grey-scale image. In the discrete form, the problem of finding the optimal layout of the structure is transformed into finding the optimal image representation of the geometry in the fixed reference domain. This fixed region, called the extended design domain, can accommodate boundary conditions and the forcing terms of the corresponding physic problem. The benefit associated with this approach is that the finite element model is not changed during the optimization process, which makes the sensitivity

\footnotetext{
${ }^{*}$ Correspondence to: Bing-Chung Chen, Department of Mechanical Engineering and Applied Mechanics, The University of Michigan, Ann Arbor, MI 48109-2125, U.S.A.

†E-mail: me-www@umich.edu
}

Contract/grant sponsor: DARPA; contract/grant number: DARPA/NIST F000765

Copyright (c) 2001 John Wiley \& Sons, Ltd. 


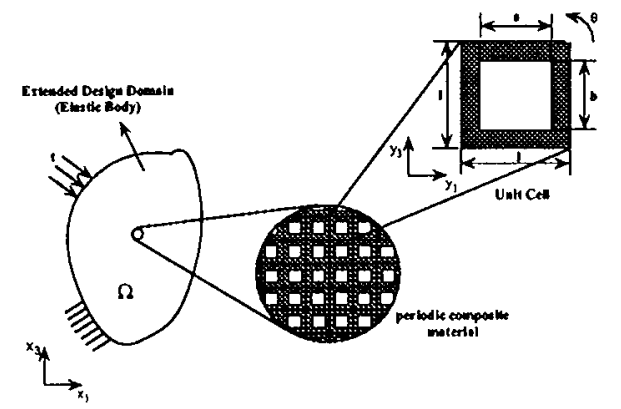

Figure 1. Relaxation of the design domain using a microstructure.

calculation easier since the boundaries of the structure are fixed throughout the optimization process.

The most straightforward image-based representation of a structural geometry is the ' $0-1$ ' integer problem where the design domain is represented by either void or full solid material. However, this formulation is not well-posed mathematically. This problem can become well posed by incorporating microstructures into the extended design domain yielding a set of continuous design variables which allow materials with intermediate properties, not only zero or full materials. This concept is called relaxation [3,4]. Several different material models have been proposed for relaxation. The microstructure proposed by Bendsøe and Kikuchi [5] consists of a square unit cell with a rectangular hole inside which its dimensions are defined by design variables $a$ and $b$, and orientation $\theta$, as shown in Figure 1. Therefore, in each point of the domain, there is a composite material defined by the periodic repetition of the microstructure corresponding to that point. In this sense, the problem consists of optimizing the material distribution in a perforated domain with infinite micro-scale voids. The effective elasticity properties of this composite material in each point of the design domain is obtained using the homogenization method described in Section 6. Alternatively, a material model using only a single variable, $\rho$, to represent the material properties is also popular. This material law models an 'artificial' isotropic material and is named solid isotropic material with penalization (SIMP) [6]. Scalar variable $\rho$ can be physically interpreted as the density of the material whose properties are in proportion to $\rho^{p}$. The introduction of these material models is justified as the final design is either entirely solid or entirely void (black-and-white design).

In this paper, we will highlight the current research which employs this material distribution paradigm to find the optimal configuration of various smart structures and microstructures. Specifically, we shall apply the topology optimization methodology to design actuators, different compliant mechanisms, and porous material microstructures with unusual thermoelastic properties. To this end, we will formulate the problem as a multi-objective optimization problem and use sequential linear programming (SLP) method to find the optimal design.

This paper is organized as follows: The optimization problem for general compliant mechanisms will be formulated in Section 2. A specialized compliant mechanism actuated by hydrostatic pressure will be introduced in Section 3. In Section 4, we will design a compliant mechanism actuated by electrical charge. This mechanism, called flextensional actuators, is a coupled structure with piezoceramics. Extension based on the compliant mechanism design 
principle will be made. In Section 5, we will use the same material distribution paradigm in the structural topology optimization to design material microstructures. Several materials microstructures with unusual thermoelastic properties will be presented.

\section{DESIGN OF COMPLIANT MECHANISMS}

Compliant mechanisms are monolithic mechanical structures that rely on elastic deformation to generate sophisticated mechanical functions. The structure is designed to be flexible in order to achieve a specified motion and to function as a mechanism. These kind of mechanisms have advantages not found in their rigid-body counterparts. Since they are basically jointless, they do not need assembly in the manufacturing process and require fewer parts. As a result, they have reduced friction, wear, backlash, and noise [7].

Recently, the compliant mechanism has received much attention since it is conceptually compatible with the micro-electro-mechanical system (MEMS) [8-10]. Micromechanical systems are built on a very small scale so it is difficult to build individual parts and use hinges or joints to assemble them as in the macro-scale manufacturing process. Designing micromechanical devices as compliant mechanisms is an easy solution for the manufacturing of MEMS since they can usually be built in a single piece and require fewer fabrication steps.

In this section, we will formulate the optimization problem to design the topology of a compliant mechanism. This formulation is based on the topology optimization techniques to find a structure which will yield maximum output displacement of the specified point in a specified direction under a given input force. Then we will extend this formulation to design compliant mechanisms which are actuated by hydrostatic pressure instead of a fixed input force. In the next section, this formulation will be extended one step further to design coupled structures with piezoceramics, called flextensional actuators, which are actuated by electrical charge.

\subsection{The formulation of mutual mean compliance}

For the first load case ( $\mathrm{LC}=1$ ), considering an elastic body, $\Omega$, subject to traction force $\mathbf{t}_{1}$ applied to boundary $\Gamma_{\mathbf{t}_{1}}$, the equilibrium equation is

$$
\int_{\Omega} \boldsymbol{\varepsilon}\left(\mathbf{v}_{1}\right)^{\mathrm{t}} \mathbf{c}^{\mathrm{E}} \boldsymbol{\varepsilon}\left(\mathbf{u}_{1}\right) \mathrm{d} \Omega=\int_{\Gamma_{\mathrm{t}}} \mathbf{t}_{1} \cdot \mathbf{v}_{1} \mathrm{~d} \Gamma, \quad \forall \mathbf{v}_{1} \in V_{0}
$$

where $\mathbf{c}^{\mathrm{E}}$ is the elastic tensor; $\varepsilon_{i j}(\mathbf{u})=\frac{1}{2}\left(\partial u_{i} / \partial x_{j}+\partial u_{j} / \partial x_{i}\right)$ is the symmetric gradient operator; $V_{0}=\left\{\mathbf{v}=v_{i} \overline{\mathbf{e}}_{i}, \quad v_{i} \in H^{1}(\Omega)\right.$ with $\mathbf{v}=0$ on $\left.\Gamma_{\mathbf{u}} i=1,2,3\right\}$ is the admissible displacement space; $\mathbf{u}_{1} \in V_{0}$ is the displacement field for $\mathrm{LC}=1$ as shown in Figure 2. By introducing the following energy bi-linear and load linear form,

$$
A(\mathbf{u}, \mathbf{v})=\int_{\Omega} \boldsymbol{\varepsilon}(\mathbf{u})^{\mathrm{t}} \mathbf{c}^{\mathrm{E}} \boldsymbol{\varepsilon}(\mathbf{v}) \mathrm{d} \Omega, \quad L_{t}(\mathbf{t}, \mathbf{v})=\int_{\Gamma_{\mathbf{t}}} \mathbf{t} \cdot \mathbf{v} \mathrm{d} \Gamma
$$

we can write Equation (1) in a compact notation

$$
A\left(\mathbf{v}_{1}, \mathbf{u}_{1}\right)=L_{t}\left(\mathbf{t}_{1}, \mathbf{v}_{1}\right) \quad \forall \mathbf{v}_{1} \in V_{0}
$$



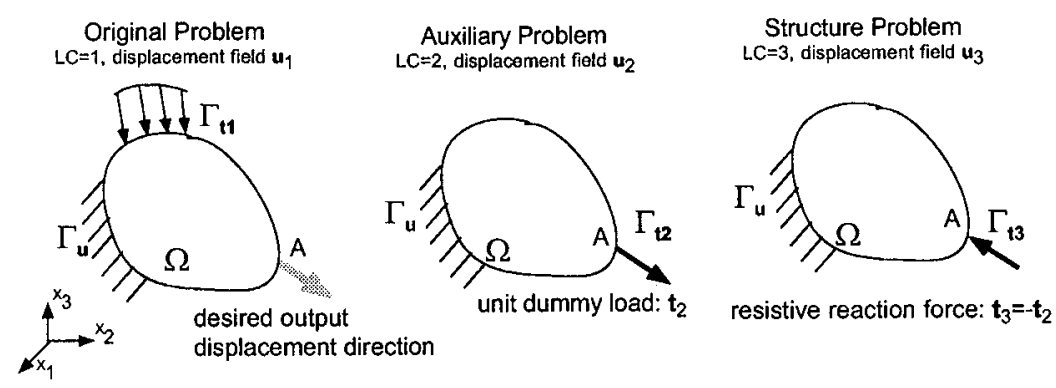

Figure 2. Two sub-programs and the structure problem in the compliant mechanism problem formulation.

Then we consider an auxiliary problem, the second load case $(\mathrm{LC}=2)$, subject to unit dummy load $\mathbf{t}_{2}$ in the direction of the desired output displacement, applied at specified point $A$. The equilibrium equation for $\mathrm{LC}=2$ in the compact notation is

$$
A\left(\mathbf{v}_{2}, \mathbf{u}_{2}\right)=L_{t}\left(\mathbf{t}_{2}, \mathbf{v}_{2}\right) \quad \forall \mathbf{v}_{2} \in V_{0}
$$

where $\mathbf{u}_{2} \in V_{0}$ is the displacement field for $L C=2$. Since $\mathbf{v}_{1}, \mathbf{v}_{2} \in V_{0}$ are arbitrary, one can substitute $\mathbf{v}_{1}$ for $\mathbf{u}_{2}$ in Equation (2) and $\mathbf{v}_{2}$ for $\mathbf{u}_{1}$ Equation (3). Furthermore, by employing the symmetry property of the elastic tensor, one can apply the reciprocal theorem and get the mutual mean compliance:

$$
L_{t}\left(\mathbf{t}_{2}, \mathbf{u}_{1}\right)=A\left(\mathbf{u}_{1}, \mathbf{u}_{2}\right)=A\left(\mathbf{u}_{2}, \mathbf{u}_{1}\right)
$$

The mutual mean compliance, $L_{t}\left(\mathbf{t}_{2}, \mathbf{u}_{1}\right)$, disguised in the energy form, is actually a projection of displacement field $\mathbf{u}_{1}$ in the direction of $\mathbf{t}_{2}$ at the point where the dummy load is applied. If one can denote this point load with the Dirac delta function at load application point $\mathbf{A}$ in the direction of $\mathbf{n}$, namely $\mathbf{t}_{2}=\delta_{\mathbf{A}}(\mathbf{x}) \mathbf{n}$, the mutual mean compliance becomes the displacement at point $\mathbf{A}$ projected in the direction of $\mathbf{n}$ :

$$
L_{t}\left(\mathbf{t}_{2}, \mathbf{u}_{1}\right)=\int_{\Gamma_{\mathbf{t}_{2}}}\left(\delta_{A}(\mathbf{x}) \mathbf{n}\right) \cdot \mathbf{u}_{1} \mathrm{~d} \Gamma=\left.\mathbf{n} \cdot \mathbf{u}_{1}(\mathbf{x})\right|_{\mathbf{x}=\mathbf{A}}
$$

If this quantity is maximized, the displacement at the point of interest specified by the dummy load $\mathbf{t}_{2}$ should be maximized when the structure is subject to load $\mathbf{t}_{1}$. Mathematically, this problem can be state as

$$
\begin{array}{lll}
\text { Maximize } & \Psi_{\mathrm{MMC}}=L_{t}\left(\mathbf{t}_{2}, \mathbf{u}_{1}\right)=\int_{\Gamma_{\mathbf{t}_{2}}} \mathbf{t}_{2} \cdot \mathbf{u}_{1} \mathrm{~d} \Gamma \\
\text { Subject to } & A\left(\mathbf{v}_{1}, \mathbf{u}_{1}\right)=L_{t}\left(\mathbf{t}_{1}, \mathbf{v}_{1}\right) \quad \forall \mathbf{v}_{1} \in V_{0} \\
& A\left(\mathbf{v}_{2}, \mathbf{u}_{2}\right)=L_{t}\left(\mathbf{t}_{2}, \mathbf{v}_{2}\right) \quad \forall \mathbf{v}_{2} \in V_{0} \\
& \text { volume constraint } &
\end{array}
$$

However, if we consider only the maximization of the mutual mean compliance, this problem is not well posed. An implementation of this objective function results in a structure without significant stiffness, having no definite shape and profile thus failing to perform the task of transforming the input force into the specified output displacement. Therefore, an objective 
function incorporating the structural functionality must be defined to provide sufficient stiffness at the region of specified output displacement. Furthermore, the structure must produce a large enough generative force and resistive reaction forces imposed by the objects the actuator intends to move or hold. This goal can be achieved by solving an optimization problem which minimizes the mean compliance of the structure subject to the resistive force at the contact region imposed by the object to be moved or held. The mean compliance, widely adopted in the topology optimization literature, is defined as the work done by the external load:

$$
L_{t}\left(\mathbf{t}_{3}, \mathbf{u}_{3}\right)=\int_{\Gamma_{\mathbf{t}_{3}}} \mathbf{t}_{3} \cdot \mathbf{u}_{3} \mathrm{~d} \Gamma
$$

This energy quantity is a global measure of the structural stiffness. By minimizing this quantity, one can find the stiffest structure under a given load.

Similarly, we may need to consider the structural stiffness at the region of input force to ensure the mechanism can maintain its shape when it deforms. This requirement can be dealt with by considering the mean compliance for the first load case,

$$
L_{t}\left(\mathbf{t}_{1}, \mathbf{u}_{1}\right)=\int_{\Gamma_{\mathbf{t}_{1}}} \mathbf{t}_{1} \cdot \mathbf{u}_{1} \mathrm{~d} \Gamma
$$

The problem of finding the stiffest structure at both the input and output regions can be formulated as a composite mean compliance function,

$$
\Psi_{\mathrm{MC}}=\alpha L_{t}\left(\mathbf{t}_{3}, \mathbf{u}_{3}\right)+(1-\alpha) L_{t}\left(\mathbf{t}_{1}, \mathbf{u}_{1}\right), \quad 0 \leqslant \alpha \leqslant 1
$$

and the optimization problem is

$$
\begin{array}{lll}
\text { Minimize } & \Psi_{\mathrm{MC}} & \\
\text { subject to } & \mathbf{t}_{3}=-\mathbf{t}_{2} \quad\left(\Gamma_{\mathbf{t}_{3}}=\Gamma_{\mathbf{t}_{2}}\right) & \\
& 0 \leqslant \alpha \leqslant 1 & \\
& A\left(\mathbf{v}_{1}, \mathbf{u}_{1}\right)=L_{t}\left(\mathbf{t}_{1}, \mathbf{v}_{1}\right) \quad \forall \mathbf{v}_{1} \in V_{0} \\
& A\left(\mathbf{v}_{3}, \mathbf{u}_{3}\right)=L_{t}\left(\mathbf{t}_{3}, \mathbf{v}_{3}\right) \quad \forall \mathbf{v}_{3} \in V_{0} \\
& \text { volume constraint } &
\end{array}
$$

To design a compliant mechanism which fulfill these conflicting design objectives, a compromising solution based on multi-criteria optimization is proposed:

$$
\begin{array}{lll}
\text { Maximize } & F \\
\text { subject to } & A\left(\mathbf{v}_{1}, \mathbf{u}_{1}\right)=L_{t}\left(\mathbf{t}_{1}, \mathbf{v}_{1}\right) & \forall \mathbf{v}_{1} \in V_{0} \\
& A\left(\mathbf{v}_{2}, \mathbf{u}_{2}\right)=L_{t}\left(\mathbf{t}_{2}, \mathbf{v}_{2}\right) & \forall \mathbf{v}_{2} \in V_{0} \\
& A\left(\mathbf{v}_{3}, \mathbf{u}_{3}\right)=L_{t}\left(\mathbf{t}_{3}, \mathbf{v}_{3}\right) & \forall \mathbf{v}_{3} \in V_{0} \\
& \mathbf{t}_{3}=-\mathbf{t}_{2} \quad\left(\Gamma_{\mathbf{t}_{3}}=\Gamma_{\mathbf{t}_{2}}\right) & \\
& \text { volume constraint } &
\end{array}
$$




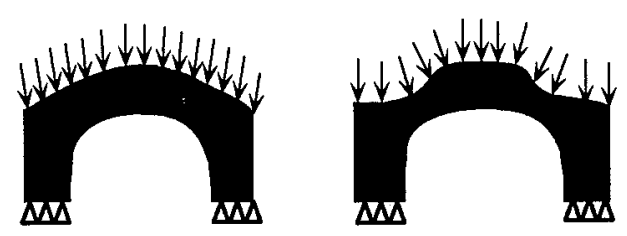

Figure 3. Hydrostatic pressure: design-dependent load.

where the objective function $F$ is defined as

$$
F=\frac{\Psi_{\mathrm{MMC}}}{\Psi_{\mathrm{MC}}}=\frac{L_{t}\left(\mathbf{t}_{2}, \mathbf{u}_{1}\right)}{\alpha L_{t}\left(\mathbf{t}_{3}, \mathbf{u}_{3}\right)+(1-\alpha) L_{t}\left(\mathbf{t}_{1}, \mathbf{u}_{1}\right)}, \quad 0 \leqslant \alpha \leqslant 1
$$

If one wants to control the contribution of the composite mean compliance, Equation (6), and mutual mean compliance, Equation (4), the following objective function is proposed:

$$
F=w \ln \left(\Psi_{\mathrm{MMC}}\right)-(1-w) \ln \left(\Psi_{\mathrm{MC}}\right), \quad 0 \leqslant w \leqslant 1
$$

where $\alpha$ and $w$ are weight coefficients to adjust the contribution of each participating objective function.

\section{DESIGN OF COMPLIANT MECHANISM ACTUATED BY HYDROSTATIC PRESSURE}

In this section, we will formulate the optimal design of the compliance mechanism actuated by hydrostatic pressure. The hydrostatic pressure forces act perpendicular to the surface of the outer boundary of the structure and are classified as design-dependent loads since they may alter their direction and location of application while the shape of the structure changes as illustrated in Figure 3.

It is not hard to find applications of the design-dependent load in many engineering disciplines. The hydrostatic pressure acting on the dam or the underwater container and the weight of snow acting on the roof are typical examples of such design-dependent loads. In a wider perspective, all structures involving solid and fluid interaction, including ducts, pipes, and airfoils, carry such design-dependent loads.

Finding an efficient algorithm to deal with design-dependent loads for structural topology optimization problems has troubled researchers in this field ever since the seminal paper by Bendsøe and Kikuchi [5] a decade ago. The underlying scheme of topology optimization originally proposed by Bendsøe and Kikuchi is based on a specific choice of a fixed-grid region upon which the optimal material distribution is to be found. This fixed region, called the extended design domain, on which the loads and constraints are specified, should be properly chosen to accommodate the loads and constraints as shown in Figure 4(a). With this approach, one can successfully find the optimal structure subject to the fixed load as illustrated in Figure 4(b) while failing to design a structure subject to the design-dependent load because the loaded surface changes as shown in Figure 4(c). The fixed-grid paradigm, though elegant and compositionally efficient for numerical implementation, has been regarded as insufficient to address the problems involving design-dependent loads such as hydrostatic 


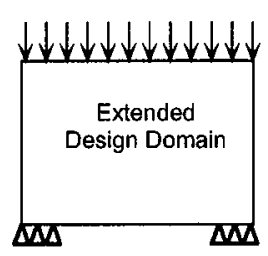

(a)

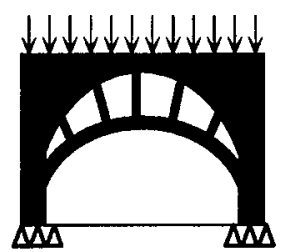

(b)

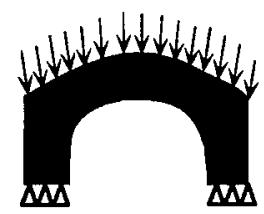

(c)

Figure 4. The structural topology optimization subject to the fixed and design-dependent loads: (a) shows the fixed extended design domain with the boundary condition and the fixed load; (b) is a possible optimal topology for a fixed loading; (c) is a possible optimal design where the pressure is allowed to change the direction and location as the loaded surface changes.

pressure. Some strategies to address this problem have been proposed. Bendsøe [11] proposed to simultaneously design the structural topology and shape of the boundary which carries the pressure loads. This approach is not efficient enough since it requires imbedding re-meshing capacities into the analysis code and complicated sensitivity analysis [12] for the optimization. Hammer and Olhoff [13] retained the fixed-grid scheme to avoid the complexity inherited from the domain shape change but used a different approach to deal with the pressure-loaded surface. A smooth surface was extracted from the iso-volumetric density curve on which the pressure acts. This approach does not need re-meshing during the analysis process, but still suffers from a rather involved sensitivity analysis for the optimization.

We proposed a new approach to simulate design-dependent loads within the context of the classical topology optimization fixed-grid paradigm [14]. The fixed-grid scheme is retained to reduce the computational complexity associated with the domain shape change. The design-dependent loads are simulated by the fictitious thermal loads as a result of a mismatch of thermal expansion coefficients among the constituent phases. The topology optimization problem is transformed into a three-phase material distribution problem within a design domain in which the solid, void, and hydrostatic fluid phases are optimally distributed.

\subsection{Formulation of design-dependent load}

Let $\Omega$ be the design domain composed of three distinctive regions, the solid $\Omega_{\mathrm{s}}$, the void $\Omega_{\mathrm{v}}$, and the fluid $\Omega_{\mathrm{f}}$. Each region is non-overlapping, i.e. $\Omega=\Omega_{\mathrm{s}} \cup \Omega_{\mathrm{v}} \cup \Omega_{\mathrm{f}}, \Omega_{\mathrm{s}} \cap \Omega_{\mathrm{v}}=\emptyset$, $\Omega_{\mathrm{s}} \cap \Omega_{\mathrm{f}}=\emptyset$, and $\Omega_{\mathrm{f}} \cap \Omega_{\mathrm{v}}=\emptyset$. The material properties are homogeneous within each region. The fluid exerts hydrostatic pressure $p$ on the solid region along interface $\Gamma_{p}$ as shown in Figure 5(a).

The equilibrium equation written in the variational form is

$$
A(\mathbf{v}, \mathbf{u})=L_{t}(\mathbf{p}, \mathbf{v}) \quad \forall \mathbf{v} \in V_{0}(\Omega)
$$

with the load linear form due to hydrostatic pressure $\mathbf{p}$

$$
L_{t}(\mathbf{p}, \mathbf{v})=\int_{\Gamma_{\mathbf{p}}}-(p \mathbf{n}) \cdot \mathbf{v} \mathrm{d} \Gamma
$$




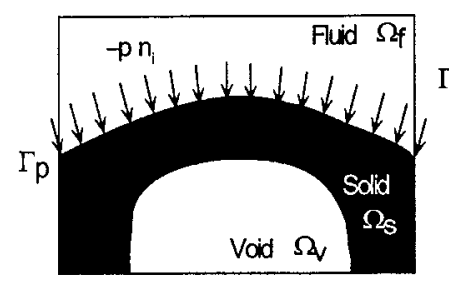

(a)

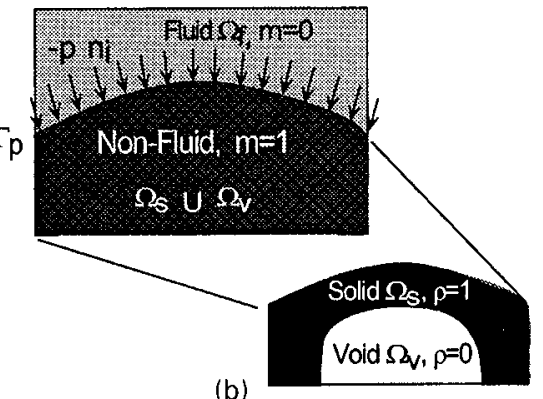

(b)

Figure 5. Schematics of the pressure loading on a structure: (a) three-phase material distribution; (b) three-phase decomposition. The domain is composed of fluid and non-fluid regions, exerting pressure at the interface. The non-fluid region is further decomposed into solid and void as in the classical two-phase topology optimization.

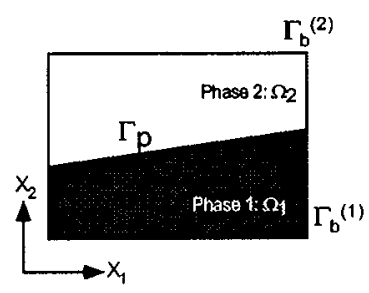

(a)

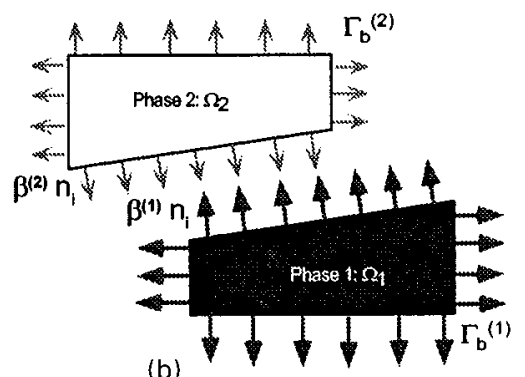

(b)

Figure 6. The loads acting on phases 1 and 2 regions when subject to the unit temperature rise: (a) two homogeneous regions with the common interface $\Gamma_{p}$ and the free boundaries $\Gamma_{\mathrm{b}}^{(1)}$ and $\Gamma_{\mathrm{b}}^{(2)}$; (b) thermal forces acting on the free boundaries and the common interface.

where $\mathbf{n}$ is the unit outward normal vector of interface $\Gamma_{p}$ and $(-p \mathbf{n})$ is the hydrostatic pressure force acting perpendicular to surface $\Gamma_{p}$. The goal is to simulate this pressure force by a fictitious thermal load. The hydrostatic pressure force is simulated by the thermal load due to a mismatch of the thermal expansion coefficients of two materials along the material interface.

Let domain $\Omega$ be composed of two regions, region $1 \Omega_{1}$ and region $2 \Omega_{2}$, made of homogeneous material phases 1 and 2, respectively. Region 1 joins region 2 on common interface $\Gamma_{p}$ with unit outward normal vector $\mathbf{n}$. Phase 1 and phase 2 regions have free boundary $\Gamma_{\mathrm{b}}^{(1)}$ and $\Gamma_{\mathrm{b}}^{(2)}$, respectively as shown in Figure $6(\mathrm{a})$. Thermal stress tensors $\beta^{(1)}$ and $\beta^{(2)}$ are homogeneous in each region where $\beta^{(1)}=\mathbf{c}^{(1)}: \alpha^{(1)}, \beta^{(2)}=\mathbf{c}^{(2)}: \alpha^{(2)}$ and $\mathbf{c}^{(1)}, \alpha^{(1)}, \mathbf{c}^{(2)}, \alpha^{(2)}$ are elastic and thermal expansion coefficient tensors of each phase, respectively. The thermal virtual work associated with the uniform unit temperature rise can be expressed as

$$
\int_{\Omega} v_{i, j} \beta_{i j} \mathrm{~d} \Omega=\int_{\Omega_{1}} v_{i, j} \beta_{i j}^{(1)} \mathrm{d} \Omega+\int_{\Omega_{2}} v_{i, j} \beta_{i j}^{(2)} \mathrm{d} \Omega
$$


Applying the Gauss divergence theorem to each region individually, one can rewrite the thermal virtual work on the homogeneous region 1 as

$$
\int_{\Omega_{1}} v_{i, j} \beta_{i j}^{(1)} \mathrm{d} \Omega=\int_{\Gamma_{\mathrm{b}}^{(1)}} v_{i} \beta_{i j}^{(1)} n_{j} \mathrm{~d} S+\int_{\Gamma_{p}} v_{i} \beta_{i j}^{(1)} n_{j} \mathrm{~d} S-\int_{\Omega_{1}} v_{i} \beta_{i j, j}^{(1)} \mathrm{d} \Omega
$$

The last term vanishes due to homogeneity. Similarly, one can transform the virtual work on region 2. Then Equation (10) can be simplified by the relation that the outward normal $\mathbf{n}$ of interface $\Gamma_{p}$ in phase 2 region point in the opposite direction as in region 1:

$$
\int_{\Omega} v_{i, j} \beta_{i j} \mathrm{~d} \Omega=\int_{\Gamma_{\mathrm{b}}^{(1)}} v_{i} \beta_{i j}^{(1)} n_{j} \mathrm{~d} S+\int_{\Gamma_{\mathrm{b}}^{(2)}} v_{i} \beta_{i j}^{(2)} n_{j} \mathrm{~d} S+\int_{\Gamma_{p}} v_{i}\left(\beta_{i j}^{(1)}-\beta_{i j}^{(2)}\right) n_{j} \mathrm{~d} S
$$

The first two terms are due to the thermal force acting on the free boundary and the last term derives from the mismatch of thermal stress tensors along interface $\Gamma_{p}$ as shown in Figure 6(b). By manipulating terms in Equation (11), one can define the 'pseudo'-linear form $L_{p}(\mathbf{v})$ as the virtual work due to thermal load, subtracting the virtual work done by the forces acting on the free boundary as

$$
\begin{aligned}
L_{p}(\mathbf{v}) & =\int_{\Omega} v_{i, j} \beta_{i j} \mathrm{~d} \Omega-\left(\int_{\Gamma_{\mathrm{b}}^{(1)}} v_{i} \beta_{i j}^{(1)} n_{j} \mathrm{~d} S+\int_{\Gamma_{\mathrm{b}}^{(2)}} v_{i} \beta_{i j}^{(2)} n_{j} \mathrm{~d} S\right) \\
& =\int_{\Gamma_{p}} v_{i}\left(\beta_{i j}^{(1)}-\beta_{i j}^{(2)}\right) n_{j} \mathrm{~d} S
\end{aligned}
$$

For a special case, when both the $\beta^{(1)}$ and $\beta^{(2)}$ are isotropic, namely, $\beta_{i j}^{(1)}=\beta^{(1)} \delta_{i j}, \beta_{i j}^{(2)}=\beta^{(2)} \delta_{i j}$, and $\beta^{(2)}$ and $\beta^{(1)}$ differ by the amount of $p$, the pseudo-linear form $L_{p}(\mathbf{v})$ is identical with the linear form $L_{t}(\mathbf{p}, \mathbf{v})$ as in Equation (9):

$$
L_{p}(\mathbf{v})=\int_{\Gamma_{p}} v_{i}\left(\beta^{(1)}-\beta^{(2)}\right) n_{i} \mathrm{~d} S=\int_{\Gamma_{p}} v_{i}\left(-p n_{i}\right) \mathrm{d} S=L_{t}(\mathbf{p}, \mathbf{v})
$$

\subsection{Material model}

We will extend this two-phase result to the three-phase system as in Figure 5(a) by adopting an engineered material model. An extended material model based on the SIMP [6] is used to simulate the three-phase material distribution. Since we want to simulate the hydrostatic pressure, the thermal stress tensors have to be isotropic. As a result, we favour this isotropic model over the orthotropic material model of microscopic square cell with a rectangular hole as shown in Figure 1.

Two sets of material models for two different regions, namely the non-fluid and fluid regions are devised. Since hydrostatic pressure forces act only at the interface between the solid and fluid regions and no interface forces exist between the solid and void regions, the non-fluid region should be free of interface force. As a result, throughout this region one needs to set the $\beta$ constant regardless of the density distribution.

In the non-fluid region, an artificial mixing assumption for the local elastic tensor, $\mathbf{c}_{e}^{(\mathrm{NF})}$ and thermal stress tensors $\beta_{e}^{(\mathrm{NF})}$ for the element $e$ of the finite element model are 
stated as:

$$
\mathbf{c}_{e}^{(\mathrm{NF})}\left(d_{e}\right)=\left(d_{e}\right)^{p} \mathbf{c}^{(s)}, \quad \beta_{e}^{(\mathrm{NF})}\left(d_{e}\right)=\beta^{(s)}
$$

In the fluid region, the classical SIMP is employed to simulate the local thermal stress tensor as

$$
\mathbf{c}_{e}^{(\mathrm{F})}\left(d_{e}\right)=\left(d_{e}\right)^{p} \mathbf{c}^{(s)}, \quad \beta_{e}^{(\mathrm{F})}\left(d_{e}\right)=\left(d_{e}\right)^{p} \beta^{(s)}
$$

where $\mathbf{c}^{(s)}$ and $\beta^{(s)}$ are elastic and thermal stress tensors of the base solid material. The local density $d_{e} \in\left[d_{\min }, 1\right]$ is assumed to be constant for given element $e$. The element is regarded as a full solid material with $d_{e}=1$ while it denotes void for $d_{e}=d_{\min }>0$. The small number, $d_{\min }$, is used to prevent singularity in the finite element calculation.

An additional parameter is necessary to distinguish between the fluid and non-fluid region. 'Dryness coefficient' $m_{e}$ for each finite element is introduced for this purpose. Those elements free from fluid entrenchment are designated with $m_{e}=1$, regardless of their local density, $d_{e}$, while the fluid elements are distinguished with $m_{e}=0$. It should be emphasized that this coefficient is used primarily to track the fluid and solid interface and is not a design variable in its own right. Coefficient $m_{e}$ for each finite element can be regarded as an implicit function of its own local density, $d_{e}$, and all the coefficients $m_{e}$ of its adjacent elements. An algorithm to determine this coefficient will be described in detail later.

Incorporating the material law in the fluid and non-fluid region in Equations (14) and (13) into a unified formula, the local material properties in element $e$ can be written as functions of coefficients $d_{e}$ in $m_{e}$ :

$$
\begin{aligned}
& \mathbf{c}_{e}\left(d_{e}, m_{e}\right)=\left(d_{e}\right)^{p} \mathbf{c}^{(s)} \\
& \beta_{e}\left(d_{e}, m_{e}\right)=m_{e} \beta_{e}^{(\mathrm{NF})}+\left(1-m_{e}\right) \beta_{e}^{(\mathrm{F})}
\end{aligned}
$$

where the thermal stress tensor $\beta_{e}$ is the arithmetic mean of fluid $\beta_{e}^{(\mathrm{F})}$ and non-fluid $\beta^{(\mathrm{NF})}$.

\subsection{Compliant mechanism actuated by hydrostatic pressure}

We will extend the problem formulation developed for the compliant mechanism, Equation (7), to a more general case when the input loads are design-dependent hydrostatic pressure as illustrated in Figure 7. Linear load form $L_{t}\left(\mathbf{t}_{1}, \mathbf{v}_{1}\right)$ for the first load case $(\mathrm{LC}=1)$ is replaced by pseudo-linear form $L_{p}\left(\mathbf{v}_{1}\right)$ in Equation (12). Since we want to design a mechanism stiff enough to sustain the input pressure load, the following optimization problem is formulated to find the stiffest structure subject to hydrostatic pressure $\mathbf{p}$ :

$$
\begin{array}{ll}
\text { Minimize } & L_{p}\left(\mathbf{u}_{1}\right) \\
\text { subject to } & A\left(\mathbf{v}_{1}, \mathbf{u}_{1}\right)=L_{p}\left(\mathbf{v}_{1}\right) \quad \forall \mathbf{v}_{1} \in V_{0} \\
& \text { volume constraint }
\end{array}
$$

Once we find the topology of this optimization problem, the resulting structural layout is employed as an initial guess for optimization problem, Eq. (7), to find the optimal topology of the compliant mechanism actuated by hydrostatic pressure. The composite mean compliance function, Equation (6), is modified to accommodate the fact that the pressure loads for $\mathrm{LC}=1$ 

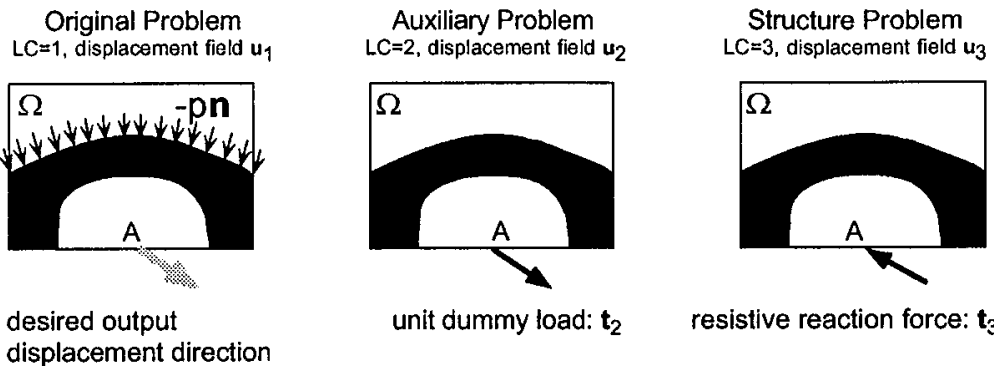
displacement direction

Figure 7. Problem formulation for the compliant mechanism actuated by hydrostatic pressure forces.

and the resistive force for $\mathrm{LC}=3$ are of different orders. In addition, as the optimization iteration process unfolds, resulting in direction and location change of the pressure loads, the order discrepancy for these two sets of loads changes accordingly. The modified composite mean compliance function is

$$
\Psi_{\mathrm{MC}}^{\prime}=(\alpha)(s) L_{t}\left(\mathbf{t}_{3}, \mathbf{u}_{3}\right)+(1-\alpha) L_{p}\left(\mathbf{u}_{1}\right)
$$

where scaling factor $s$ is the ratio of the $L_{\infty}$ norms of vector of the element strain energy $\left(\mathbf{A}^{e}(\mathbf{u}, \mathbf{u})=\left\{A^{e}(\mathbf{u}, \mathbf{u})\right\}^{\mathrm{T}}, e=1\right.$ to $\left.\mathrm{NE}\right)$ between the two different load cases,

$$
s=\frac{\left\|\mathbf{A}^{e}\left(\mathbf{u}_{1}, \mathbf{u}_{1}\right)\right\|_{L_{\infty}}}{\left\|\mathbf{A}^{e}\left(\mathbf{u}_{3}, \mathbf{u}_{3}\right)\right\|_{L_{\infty}}}
$$

In this work, we choose the objective function $\mathscr{F}$ as

$$
\mathscr{F}=\frac{\Psi_{\mathrm{MMC}}}{\Psi_{\mathrm{MC}}^{\prime}}=\frac{L_{t}\left(\mathbf{t}_{2}, \mathbf{u}_{1}\right)}{(\alpha)(s) L_{t}\left(\mathbf{t}_{3}, \mathbf{u}_{3}\right)+(1-\alpha) L_{p}\left(\mathbf{u}_{1}\right)}
$$

and weight coefficient $\alpha=0.5$.

\subsection{Numerical example of compliant mechanism actuated by hydrostatic pressure}

This example demonstrates the applicability for sensors and actuators design in the form of the compliant mechanisms. We want to design a compliant mechanism which converts the pressure loads into the rotational motion as illustrated in Figure 8.

In the first stage, we will find the optimal topology of a structure subject to the input pressure load as formulated in Equation (15). The solid region shall occupy no more than 30 per cent of the total volume of the design domain.

The initial design domain is composed of 'fixed fluid' regions from which the fluid originates, and are not subject to change during the subsequent optimization iterations. The fluid will propagate to all the neighbouring elements unless stopped by the solid elements which the fluid cannot penetrate. The fluid propagating process is illustrated in Figure 9. By using this algorithm, one can keep track of the interface of the fluid region.

The pressure force is kept fixed during the first 25 iterations and results in a constant distributed load acting on the boundary of the fixed fluid region within the design domain. In other words, it is not until the 26th iteration that the 'flood-over' algorithm will be applied 


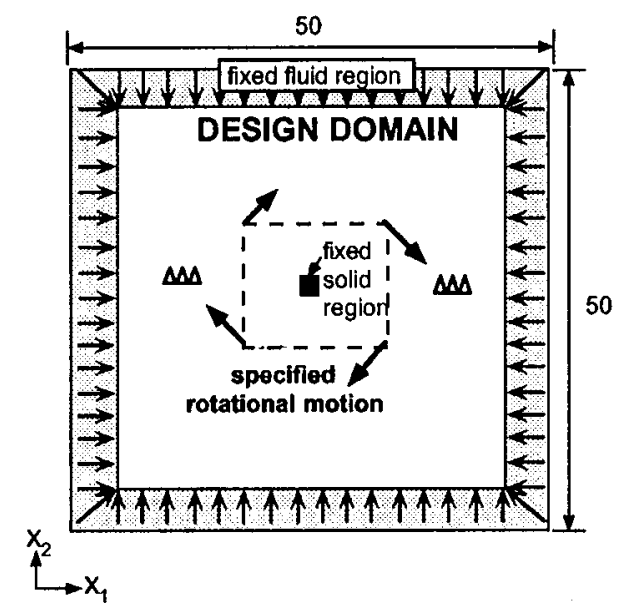

Figure 8. Design domain and the specified rotational motion.

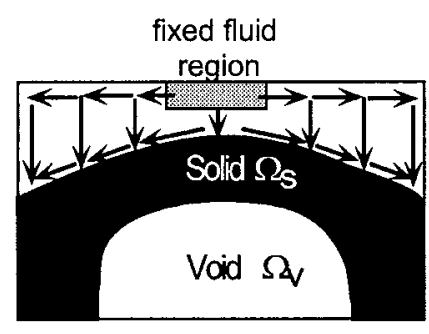

(a)

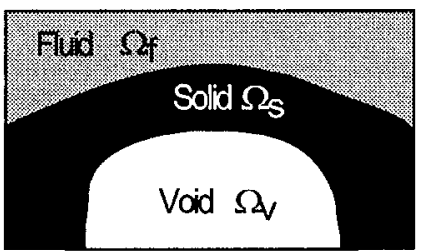

(b)

Figure 9. The schematic of the fluid flooding algorithm to track the interface of solid and fluid regions: (a) the fluid originates from the fixed fluid region and will propagate to the adjacent neighbours. This propagating process is stopped at the solid interface; (b) after the propagating process, the design domain is separated into three regions, the solid, void, and fluid.

to track the fluid interface. The primary reason for this scheme is to prevent the fluid region from flooding all over the domain, should the flood-over algorithm be applied in the first place when there is no significant structure acting as a containment for the fluid region. The resulting optimal structural topology for this stage is shown in Figure 10.

In the second stage, we find the optimal topology of the compliant mechanism which converts the pressure loads into the rotational motion. The topology found in the first stage is used as the initial guess for the second stage. The objective function is defined in Equation (17). The optimized topology of the mechanism is shown in Figure 11(a) and the deformation is shown in Figure 11(b). 


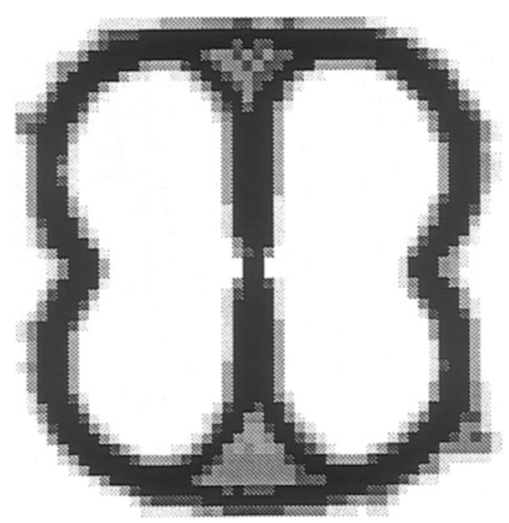

Figure 10. The optimal structural topology subject to hydrostatic pressure considering structure stiffness.

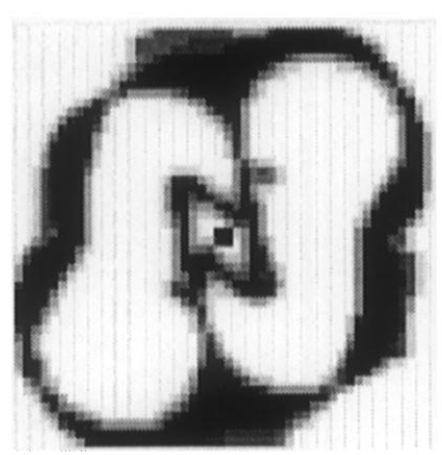

(a)

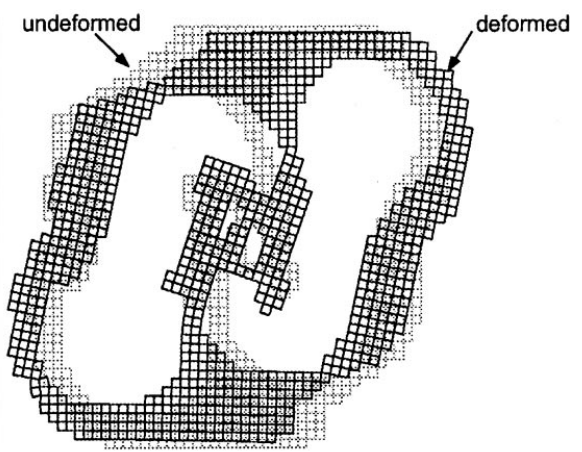

(b)

Figure 11. (a) Topology result; (b) deformed structure.

\section{DESIGN OF FLEXTENSIONAL ACTUATORS}

Piezoelectric materials have the property of converting electrical energy (electric field and electrical charge) into mechanical energy (strain and stress) and vice versa. They are widely used in electromechanical sensors and actuators, ultrasonic transducers for medical imaging and non-destructive evaluation (NDE), underwater acoustics (some hydrophones and naval sonars), and other applications. In engineering, the piezoelectric materials applied are usually ceramics (piezoceramics).

However, the displacements generated by piezoelectric materials are very small (order of nanometers) and since in most part of applications large output displacements are necessary, a flexible mechanical structure (coupling structure) is connected to the piezoceramic to amplify and convert the output piezoceramic displacements. This generates a device called flextensional piezoelectric transducer [15]. These transducers can be used to control vibration in structures, to actuate flap wings in modern airplanes, and also can be applied as sonar devices. The performance of a flextensional transducer is measured in terms of output displacement and 
generative (or 'blocking') force, and in dynamic applications the transducer must oscillate in a desired resonance frequency. Generative force is the maximum force supported by the transducer without deforming for a certain applied voltage.

Flextensional transducers have been developed by using simple analytical models and experimental techniques [16, 17], and the finite element method [18, 19] (FEM). However, the design is limited to the optimization of some dimension of a specific topology chosen for the coupling structure. These studies showed that the performance and resonance frequency depend on the distribution of mass, stiffness, and flexibility in the coupling structure domain, which is related to the coupling structure topology. Therefore, the design of the coupling structure can be achieved by using topology optimization. The nature of the design of flextensional transducers makes the application of topology optimization very attractive. By designing other types of flexible structures connected to the piezoceramic, we can obtain other types of flextensional transducers that produce high output displacements (or generative forces) in different directions, and also in a desired frequency, according to a specific application.

Based on this idea, a method for designing flextensional transducers for static and dynamic (inertia effect is considered) applications is proposed by applying topology optimization. The problem is posed as the design of a flexible structure coupled to the piezoceramic that maximizes the output displacement and generative force in a specified point of the domain and direction, in a specified frequency. The topology optimization method applied is based on the homogenization design method developed by Bendsøe and Kikuchi [5]. FEM is applied to the structural analysis in the optimization procedure. Although the method introduced is general and can be applied to three-dimensional (3D) models, the examples presented herein are limited to two-dimensional (2D plane strain) models due to lower computational cost.

\subsection{FEM piezoelectric modelling}

A general method such as the finite element method (FEM) is necessary for the structural analysis since complex topologies are expected as a topology optimization result.

The flextensional transducers considered for design operate in a frequency $\omega$. Therefore, the finite element equations for modelling a linear piezoelectric medium considering a harmonic analysis are [20]:

$$
\left\{\left[\begin{array}{ll}
\mathbf{K}_{\mathbf{u u}} & \mathbf{K}_{\mathbf{u} \phi} \\
\mathbf{K}_{\mathbf{u} \phi}^{\mathrm{t}} & \mathbf{K}_{\boldsymbol{\phi} \phi}
\end{array}\right]-\omega^{2}\left[\begin{array}{cc}
\mathbf{M} & \mathbf{0} \\
\mathbf{0} & \mathbf{0}
\end{array}\right]\right\}\left\{\begin{array}{l}
\mathbf{U} \\
\boldsymbol{\phi}
\end{array}\right\}=\left\{\begin{array}{l}
\mathbf{F} \\
\mathbf{Q}
\end{array}\right\}
$$

where $\mathbf{K}_{\mathbf{u u}}, \mathbf{K}_{\mathbf{u} \phi}, \mathbf{K}_{\phi \phi}$, and $\mathbf{M}$ are the stiffness, piezoelectric, dielectric and mass matrices, respectively, and $\mathbf{F}, \mathbf{Q}, \mathbf{U}$ and $\boldsymbol{\phi}$ are the nodal mechanical force, nodal electrical charge, nodal displacements and nodal electric potential vectors, respectively. Damping was not considered in a first implementation. For more details, refer to Reference [20].

\subsection{Formulation of the topology optimization problem}

4.2.1. Mean transduction. In the formulation of the design optimization problem of flextensional transducers, the concept of mean transduction is introduced. This concept is obtained by extending the reciprocal theorem (Betti's theorem) from elasticity theory to the piezoelectric medium. This extension is described in detail in Reference [21] and here only the final result is discussed. 
Considering a piezoelectric body, subjected to electrical charges $d_{1}, d_{2}$ applied to the boundary $\Gamma_{d_{1}}$ and $\Gamma_{d_{2}}$, respectively, and tractions $\mathbf{t}_{1}, \mathbf{t}_{2}$ applied to the boundaries $\Gamma_{\mathbf{t}_{1}}$ and $\Gamma_{\mathbf{t}_{2}}$, respectively, we can say that

$$
L_{1}\left(\mathbf{u}_{2}, \phi_{2}\right)=\int_{\Gamma_{\mathbf{t}_{1}}} \mathbf{t}_{1} \cdot \mathbf{u}_{2} \mathrm{~d} \Gamma+\int_{\Gamma_{d_{1}}} d_{1} \phi_{2} \mathrm{~d} \Gamma=\int_{\Gamma_{\mathbf{t}_{2}}} \mathbf{t}_{2} \cdot \mathbf{u}_{1} \mathrm{~d} \Gamma+\int_{\Gamma_{d_{2}}} d_{2} \phi_{1} \mathrm{~d} \Gamma=L_{2}\left(\mathbf{u}_{1}, \phi_{1}\right)
$$

where $\phi_{1}$ and $\phi_{2}$ are electric potentials. We will call $L_{1}\left(\mathbf{u}_{2}, \phi_{2}\right)$ (or $\left.L_{2}\left(\mathbf{u}_{1}, \phi_{1}\right)\right)$ mean transduction since mechanical and electrical quantities are involved.

In dynamic applications, $\mathbf{u}_{1}=\overline{\mathbf{u}}_{1} \mathrm{e}^{\mathrm{j} \omega \tau}, \quad \mathbf{u}_{2}=\overline{\mathbf{u}}_{2} \mathrm{e}^{\mathrm{j} \omega \tau}, \quad \mathbf{t}_{1}=\overline{\mathbf{t}}_{1} \mathrm{e}^{\mathrm{j} \omega \tau}, \quad \mathbf{t}_{2}=\overline{\mathbf{t}}_{2} \mathrm{e}^{\mathrm{j} \omega \tau}, \quad d_{1}=\bar{d}_{1} \mathrm{e}^{\mathrm{j} \omega \tau}$, $d_{2}=\bar{d}_{2} \mathrm{e}^{\mathrm{j} \omega \tau}, \phi_{1}=\bar{\phi}_{1} \mathrm{e}^{\mathrm{j} \omega \tau}$ and $\phi_{2}=\bar{\phi}_{2} \mathrm{e}^{\mathrm{j} \omega \tau}$, where $\omega$ stands for an excitation frequency, $\tau$ stands for time, and $\overline{\mathbf{u}}_{1}, \overline{\mathbf{u}}_{2}, \overline{\mathbf{t}}_{1}, \overline{\mathbf{t}}_{2}, \bar{d}_{1}, \bar{d}_{2}, \bar{\phi}_{1}$ and $\bar{\phi}_{2}$ are the amplitudes of $\mathbf{u}_{1}, \mathbf{u}_{2}, \mathbf{t}_{1}, \mathbf{t}_{2}, d_{1}, d_{2}, \phi_{1}$ and $\phi_{2}$, respectively. Since no damping is considered, there is no phase difference among these quantities.

Equation (20) consist of the weak formulation of the equilibrium equations of the piezoelectric medium [21]. Notice that the dependence on $\mathrm{e}^{\mathrm{j} \omega \tau}$ is not represented because it appears in both sides of the equation, and therefore it cancels out. In addition, for the sake of simplicity of notation, the amplitudes are represented without using the overbar:

$$
\begin{array}{r}
\int_{\Omega} \boldsymbol{\varepsilon}(\mathbf{u})^{\mathrm{t}} \mathbf{c}^{\mathrm{E}} \boldsymbol{\varepsilon}(\mathbf{v}) \mathrm{d} \Omega-\omega^{2} \int_{\Omega} \rho \mathbf{u}^{\mathrm{t}} \mathbf{v} \mathrm{d} \Omega+\int_{\Omega}(\nabla \phi)^{\mathrm{t}} \mathbf{e}^{\mathrm{t}} \boldsymbol{\varepsilon}(\mathbf{v}) \mathrm{d} \Omega=\int_{\Gamma_{\mathbf{t}}} \mathbf{t} \cdot \mathbf{v} \mathrm{d} \Gamma \\
\int_{\Omega} \boldsymbol{\varepsilon}(\mathbf{u})^{\mathrm{t}} \mathbf{e} \nabla \varphi \mathrm{d} \Omega-\int_{\Omega}(\nabla \phi)^{\mathrm{t}} \boldsymbol{\varepsilon}^{\mathrm{S}} \nabla \varphi \mathrm{d} \Omega=\int_{\Gamma_{d}} \mathrm{~d} \varphi \mathrm{d} \Gamma \\
\text { for } \mathbf{u}, \phi \in V \text { and } \forall \mathbf{v}, \forall \varphi \in V
\end{array}
$$

where $V=\left\{\mathbf{v}=v_{i} \overline{\mathbf{e}}_{i}, \varphi: v_{i}, \varphi \in H^{1}(\Omega)\right.$ with $\mathbf{v}=0$ on $\Gamma_{\mathbf{u}}$ and $\varphi=0$ on $\Gamma_{\phi}, i=1$ or 3$\}, \Omega$ now is the domain of the piezoelectric medium (but it may contain non-piezoelectric materials also), $\boldsymbol{\nabla}$ is the gradient operator, and $\mathbf{c}^{\mathrm{E}}$, e, and $\varepsilon^{\mathrm{S}}$ are the elastic, piezoelectric, and dielectric properties, respectively, of the medium, and $\rho$ is the density. ' $t$ ' denotes transpose, $\varepsilon_{i j}(\mathbf{u})=\frac{1}{2}\left(\partial u_{i} / \partial x_{j}+\partial u_{j} / \partial x_{i}\right)$, and $\mathbf{v}$ and $\varphi$ are virtual displacements and electric potential, respectively. The index $i$ assumes value 1 or 3 because the problem is considered in the plane $1-3$. The piezoceramic is polarized in the \#3 direction.

To write Equation (20) in a more compact form, we define the following operators:

$$
\begin{aligned}
A(\mathbf{u}, \mathbf{v}) & =\int_{\Omega} \boldsymbol{\varepsilon}(\mathbf{u})^{\mathrm{t}} \mathbf{c}^{\mathrm{E}} \boldsymbol{\varepsilon}(\mathbf{v}) \mathrm{d} \Omega, \quad B(\phi, \mathbf{v})=\int_{\Omega}(\nabla \phi)^{\mathrm{t}} \mathbf{e}^{\mathrm{t}} \boldsymbol{\varepsilon}(\mathbf{v}) \mathrm{d} \Omega \\
C(\phi, \varphi) & =\int_{\Omega}(\nabla \phi)^{\mathrm{t}} \boldsymbol{\varepsilon}^{\mathrm{S}} \nabla \varphi \mathrm{d} \Omega, \quad L_{t}(\mathbf{t}, \mathbf{v})=\int_{\Gamma_{\mathbf{t}}} \mathbf{t} \cdot \mathbf{v} \mathrm{d} \Gamma \\
L_{d}(d, \varphi) & =\int_{\Gamma_{d}} \mathrm{~d} \varphi \mathrm{d} \Gamma, \quad M(\mathbf{u}, \mathbf{v})=\int_{\Omega} \rho \mathbf{u}^{\mathrm{t}} \mathbf{v} \mathrm{d} \Omega
\end{aligned}
$$

Therefore, Equation (20) becomes

$$
\begin{aligned}
A(\mathbf{u}, \mathbf{v})-\omega^{2} M(\mathbf{u}, \mathbf{v})+B(\phi, \mathbf{v}) & =L_{t}(\mathbf{t}, \mathbf{v}) \\
B(\varphi, \mathbf{u})-C(\phi, \varphi) & =L_{d}(d, \varphi)
\end{aligned}
$$


By making $\mathbf{u}, \mathbf{v}$ equal to $\mathbf{u}_{1}$ and $\mathbf{u}_{2}, \mathbf{t}$ equal to $\mathbf{t}_{1}$ and $\mathbf{t}_{2}, d$ equal to $d_{1}$ and $d_{2}$, and $\phi, \varphi$ equal to $\phi_{1}$ and $\phi_{2}$, (for every $\mathbf{u}_{1}, \mathbf{u}_{2}, \phi_{1}$, and $\phi_{2}$ admissible), and substituting the definitions $L_{t}\left(\mathbf{t}_{1}, \mathbf{u}_{2}\right)$ and $L_{d}\left(d_{1}, \phi_{2}\right)$ into Equation (19), this equation can be written in the form

$$
\begin{aligned}
L_{1}\left(\mathbf{u}_{2}, \phi_{2}\right) & =A\left(\mathbf{u}_{1}, \mathbf{u}_{2}\right)-\omega^{2} M\left(\mathbf{u}_{1}, \mathbf{u}_{2}\right)+B\left(\phi_{1}, \mathbf{u}_{2}\right)+B\left(\phi_{2}, \mathbf{u}_{1}\right)-C\left(\phi_{1}, \phi_{2}\right) \\
& =A\left(\mathbf{u}_{2}, \mathbf{u}_{1}\right)-\omega^{2} M\left(\mathbf{u}_{2}, \mathbf{u}_{1}\right)+B\left(\phi_{2}, \mathbf{u}_{1}\right)+B\left(\phi_{1}, \mathbf{u}_{2}\right)-C\left(\phi_{2}, \phi_{1}\right) \\
& =L_{2}\left(\mathbf{u}_{1}, \phi_{1}\right)
\end{aligned}
$$

Now, applying the FEM formulation by discretizing the domain $\Omega$ (displacements and electric potentials are approximated by using shape functions), the reciprocal theorem and mean transduction for the piezoelectric medium in the dynamic case can be written in the following matrix form:

$$
\begin{aligned}
L_{1}\left(\mathbf{U}_{2}, \boldsymbol{\phi}_{2}\right) & =\left\{\mathbf{U}_{2}\right\}^{\mathrm{t}}\left\{\mathbf{F}_{1}\right\}+\left\{\boldsymbol{\phi}_{2}\right\}^{\mathrm{t}}\left\{\mathbf{Q}_{1}\right\} \\
& =\left\{\begin{array}{c}
\mathbf{U}_{2}^{\mathrm{t}} \\
\boldsymbol{\phi}_{2}^{\mathrm{t}}
\end{array}\right\}^{\mathrm{t}}\left\{\left[\begin{array}{cc}
\mathbf{K}_{\mathbf{u u}} & \mathbf{K}_{\mathbf{u \phi}} \\
\mathbf{K}_{\mathbf{u} \boldsymbol{\phi}}^{\mathrm{t}} & -\mathbf{K}_{\boldsymbol{\phi} \boldsymbol{\phi}}
\end{array}\right]-\omega^{2}\left[\begin{array}{cc}
\mathbf{M} & 0 \\
0 & 0
\end{array}\right]\right\}\left\{\begin{array}{l}
\mathbf{U}_{1} \\
\boldsymbol{\phi}_{1}
\end{array}\right\}=\left\{\mathbf{W}_{2}\right\}^{\mathrm{t}}[\overline{\mathbf{K}}]\left\{\mathbf{W}_{1}\right\} \\
& =\left\{\mathbf{W}_{1}\right\}^{\mathrm{t}}[\overline{\mathbf{K}}]\left\{\mathbf{W}_{2}\right\}=\left\{\begin{array}{c}
\mathbf{U}_{1}^{\mathrm{t}} \\
\boldsymbol{\phi}_{1}^{\mathrm{t}}
\end{array}\right\}^{\mathrm{t}}\left\{\left[\begin{array}{cc}
\mathbf{K}_{\mathbf{u u}} & \mathbf{K}_{\mathbf{u \phi}} \\
\mathbf{K}_{\mathbf{u} \boldsymbol{\phi}}^{\mathrm{t}} & -\mathbf{K}_{\boldsymbol{\phi} \boldsymbol{\phi}}
\end{array}\right]-\omega^{2}\left[\begin{array}{cc}
\mathbf{M} & 0 \\
0 & 0
\end{array}\right]\right\}\left\{\begin{array}{l}
\mathbf{U}_{2} \\
\boldsymbol{\phi}_{2}
\end{array}\right\} \\
& =\left\{\mathbf{U}_{1}\right\}^{\mathrm{t}}\left\{\mathbf{F}_{2}\right\}+\left\{\boldsymbol{\phi}_{1}\right\}^{\mathrm{t}}\left\{\mathbf{Q}_{2}\right\}=L_{2}\left(\mathbf{U}_{1}, \boldsymbol{\phi}_{1}\right)
\end{aligned}
$$

where $\mathbf{U}_{i}, \boldsymbol{\phi}_{i}, \mathbf{F}_{i}, \mathbf{Q}_{i},(i=1,2), \mathbf{K}_{\mathbf{u u}}, \mathbf{K}_{\mathbf{u} \boldsymbol{\phi}}, \mathbf{K}_{\mathbf{\phi} \boldsymbol{\phi}}$, and $\mathbf{M}$ were defined in Section 4.1. Equation (24) allows us to implement the concept of mean transduction for the piezoelectric medium in the dynamic case by using FEM formulated in a discretized domain. For static applications $\omega=0$, and inertia effects are neglected. Thus, we can express the displacement or electric potential in any region of the piezoelectric medium as a function of a known displacement and electric fields caused by an external applied dummy traction or surface charge.

4.2.2. Formulation of objective function and constraints. Two different objective functions must be considered in the flextensional transducer design [21]: mean transduction and mean compliance.

Mean transduction described in Equation (24) is related to the electromechanical conversion represented by the displacement generated in region $\Gamma_{\mathbf{t}_{2}}$ in a certain direction due to an input electrical charge in region $\Gamma_{d_{1}}$ of the design domain (see Figure 12). The larger this function, the larger the displacement generated in region $\Gamma_{\mathbf{t}_{2}}$ in the specified frequency $\omega$. The mean transduction is calculated considering the two load cases described in case 1 of Figure 12: the first one is related to the transducer response due to the application of a periodically oscillating surface charge on surface $\Gamma_{d_{1}}$ of the transducer; and the second one is related to an applied periodically oscillating dummy traction $\mathbf{t}_{2}$ to region $\Gamma_{\mathbf{t}_{2}}$, in the same desired output 

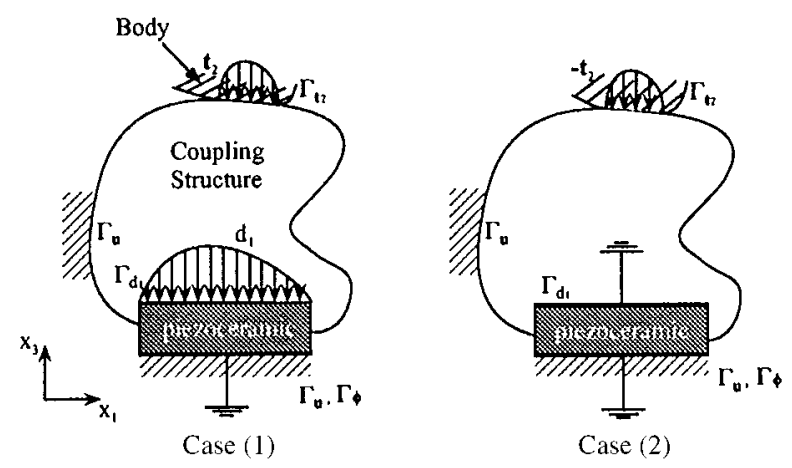

Figure 12. Load cases for calculation of mean transduction (case 1) and mean compliance (case 2).

displacement direction. Therefore, the maximization of the mean transduction is related to the maximization of the output displacement.

Considering Equation (24), since the mechanical traction is null in the first load case $\left(\mathbf{t}_{1}=0\right)$ and the surface charges are null in the second load case $\left(d_{2}=0\right)$, the mean transduction in dynamic applications between regions $\Gamma_{d_{1}}$ and $\Gamma_{\mathbf{t}_{2}}$ is given by [21]

$$
\begin{aligned}
L_{2}\left(\mathbf{u}_{1}, \phi_{1}\right) & =L_{t}\left(\mathbf{t}_{2}, \mathbf{u}_{1}\right)=L_{d}\left(d_{1}, \phi_{2}\right) \\
& =A\left(\mathbf{u}_{1}, \mathbf{u}_{2}\right)-\omega^{2} M\left(\mathbf{u}_{1}, \mathbf{u}_{2}\right)+B\left(\phi_{1}, \mathbf{u}_{2}\right)+B\left(\phi_{2}, \mathbf{u}_{1}\right)-C\left(\phi_{1}, \phi_{2}\right) \\
& =B\left(\phi_{2}, \mathbf{u}_{1},\right)-C\left(\phi_{1}, \phi_{2}\right)
\end{aligned}
$$

Note that in the dynamic case, under the harmonic excitation, the deformation in the direction of $-\mathbf{t}_{2}$, where the phase angle is $\omega \tau$, is identical to the deformation in the direction of $\mathbf{t}_{2}$, where the phase angle is $\omega \tau+\pi$. Therefore, the maximization of the absolute value of the mean transduction $\left|L_{2}\left(\mathbf{u}_{1}, \phi_{1}\right)\right|$ must be considered instead.

However, if only the maximization of the mean transduction is considered, the optimum solution obtained for this function may be a structure with very small stiffness. Therefore, a structural function must be defined to provide sufficient stiffness between $\Gamma_{\mathbf{t}_{2}}$ and $\Gamma_{d_{1}}$, which is obtained by minimizing the mean compliance between $\Gamma_{\mathbf{t}_{2}}$ and $\Gamma_{d_{1}}$. This also guarantees a large generative force, and also that the transducer will resist to reaction forces generated by some body that the transducer is trying to hold or move. The mean compliance is calculated considering a static load case described in case 2 of Figure 12 where the electrode surface $\Gamma_{d_{1}}$ is electrically grounded. Therefore, the mean compliance is given by the expression [21]

$$
\begin{aligned}
L_{3}\left(\mathbf{u}_{3}, \phi_{3}\right) & =\int_{\Gamma_{\mathbf{t}_{2}}} \mathbf{t}_{3} \cdot \mathbf{u}_{3} \mathrm{~d} \Gamma \\
& =A\left(\mathbf{u}_{3}, \mathbf{u}_{3}\right)+2 B\left(\phi_{3}, \mathbf{u}_{3}\right)-C\left(\phi_{3}, \phi_{3}\right) \\
& =A\left(\mathbf{u}_{3}, \mathbf{u}_{3}\right)+B\left(\phi_{3}, \mathbf{u}_{3}\right)
\end{aligned}
$$

since $d_{3}=0$. By changing the mean compliance value we can control the generative force. 
To combine both optimization problems, the following objective function is proposed:

$$
F=w \ln \left(\left|L_{2}\left(\mathbf{u}_{1}, \phi_{1}\right)\right|\right)-(1-w) \ln \left(L_{3}\left(\mathbf{u}_{3}, \phi_{3}\right)\right) \quad 0 \leqslant w \leqslant 1
$$

where $w$ is a weight coefficient. This objective function allows us to control the contributions of the mean transduction (25) and the mean compliance (27) in the design. Therefore, the optimization problem is stated as

$$
\begin{array}{ll}
\begin{array}{ll}
\text { Maximize } \\
a, b, \text { and } \theta
\end{array} & F \\
\text { subject to } & \mathbf{t}_{3}=-\mathbf{t}_{2} \quad\left(\Gamma_{\mathbf{t}_{3}}=\Gamma_{\mathbf{t}_{2}}\right) \\
& A\left(\mathbf{u}_{1}, \mathbf{v}_{1}\right)-\omega^{2} M\left(\mathbf{u}_{1}, \mathbf{v}_{1}\right)+B\left(\phi_{1}, \mathbf{v}_{1}\right)=0 \\
& B\left(\varphi_{1}, \mathbf{u}_{1}\right)-C\left(\phi_{1}, \varphi_{1}\right)=L_{d}\left(d_{1}, \varphi_{1}\right) \quad \text { for } \mathbf{u}_{1}, \phi_{1} \in V_{a} \text { and } \forall \mathbf{v}_{1}, \forall \varphi_{1} \in V_{a} \\
& A\left(\mathbf{u}_{2}, \mathbf{v}_{2}\right)-\omega^{2} M\left(\mathbf{u}_{2}, \mathbf{v}_{2}\right)+B\left(\phi_{2}, \mathbf{v}_{2}\right)=L_{t}\left(\mathbf{t}_{2}, \mathbf{v}_{2}\right) \\
& B\left(\varphi_{2}, \mathbf{u}_{2}\right)-C\left(\phi_{2}, \varphi_{2}\right)=0 \quad \text { for } \mathbf{u}_{2}, \phi_{2} \in V_{a} \text { and } \forall \mathbf{v}_{2}, \forall \varphi_{2} \in V_{a} \\
& A\left(\mathbf{u}_{3}, \mathbf{v}_{3}\right)+B\left(\phi_{3}, \mathbf{v}_{3}\right)=L_{t}\left(\mathbf{t}_{3}, \mathbf{v}_{3}\right) \\
& B\left(\varphi_{3}, \mathbf{u}_{3}\right)-C\left(\phi_{3}, \varphi_{3}\right)=0 \quad \text { for } \mathbf{u}_{3}, \phi_{3} \in V_{b} \text { and } \forall \mathbf{v}_{3}, \forall \varphi_{3} \in V_{b} \\
& 0 \leqslant a \leqslant a_{\text {sup }}<1 \\
& 0 \leqslant b \leqslant b_{\text {sup }}<1 \\
& \Theta(a, b)=\int(1-a b) \mathrm{d} S-\Theta_{S} \leqslant 0
\end{array}
$$

where $V_{a}=\left\{\mathbf{v}=v_{i} \overline{\mathbf{e}}_{i}, \varphi: v_{i}, \varphi \in H^{1}(\Omega)\right.$ with $\mathbf{v}=0$ on $\Gamma_{u}$ and $\varphi=0$ on $\Gamma_{\phi}, i=1$ or 3$\}$ and $V_{b}=\left\{\mathbf{v}=v_{i} \overline{\mathbf{e}}_{i}, \varphi: v_{i}, \varphi \in H^{1}(\Omega)\right.$ with $\mathbf{v}=0$ on $\Gamma_{u}$, and $\varphi=0$ on $\Gamma_{\phi}$ and $\Gamma_{d_{1}}, i=1$ or 3$\}$. $S$ is the design domain $\Omega$ excluding the piezoceramic, $\Theta$ is the volume of this design domain, and $\Theta_{S}$ is an upper-bound volume constraint that controls the maximum amount of material used to build the coupling structure. The other constraints are the equilibrium equations.

The above optimization problem was defined in a continuous form, however since the domain is discretized in $N$ finite elements, the above definitions must be substituted by their equivalent discretized ones using FEM (including the equilibrium equations). In addition, the variables $a, b$, and $\theta$ which theoretically are a continuous functions, become sets of continuous design variables $a_{n}, b_{n}$, and $\theta_{n}$ defined for the $n$ finite element subdomain in the numerical problem. The upper-bounds $a_{\text {sup }}=0.995$ and $b_{\text {sup }}=0.995$ specified for $a$ and $b$, respectively, are necessary to avoid numerical problems such as singularity of the stiffness matrix in the finite element formulation. Numerically, regions with $a_{n}=b_{n}=0.995$ have practically no structural significance and can be considered void regions.

\subsection{Numerical implementation of flextential acutators}

A flow chart of the optimization algorithm describing the steps involved is shown in Figure 13. The software was implemented in FORTRAN language.

Due to the large number of design variables the optimization problem is solved using sequential linear programming (SLP). SLP consists of the sequential solution of approximate linear subproblems that can be defined by writing a Taylor series expansion of the objective and constraint functions around the current design points $a_{n}$ and $b_{n}$ in each iteration step 


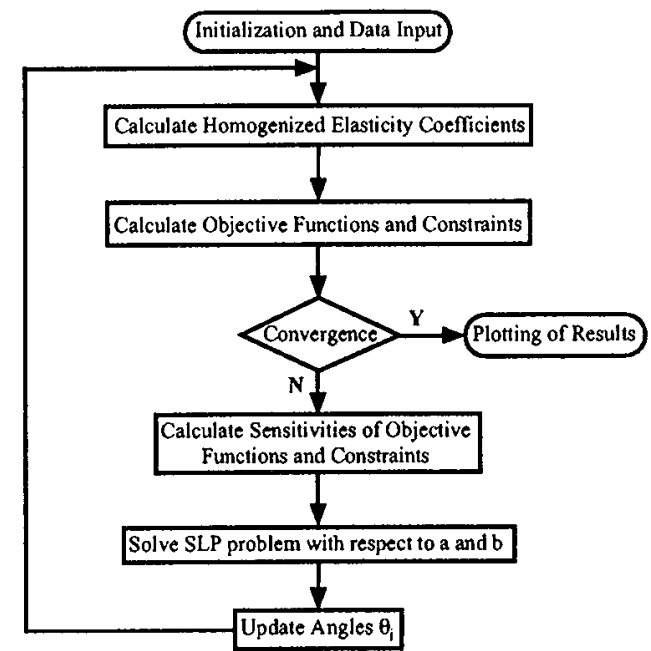

Figure 13. Flow chart of the optimization procedure.

( $\theta_{n}$ is calculated after the optimization step). The linearization of the problem (Taylor series) in each iteration requires the sensitivities (gradients) of the objective function $(F)$ and constraints in relation to design variables $a_{n}$ and $b_{n}$ [21]. These gradients depend on the gradients of the elastic properties in relation to $a$ and $b$ which can be calculated by building a table with values of homogenized elastic properties obtained for combinations of discrete values of $a$ and $b$.

In each iteration, moving limits are defined for the design variables. Typically during one iteration, the design variables will be allowed to change by 10 per cent of their original values. After optimization, a new set of design variables $a_{n}$ and $b_{n}$ is obtained and updated in the design domain. $\theta_{n}$ is obtained by considering the local principal stress directions in each finite element after each optimization step [22]. The iteration steps continue until the convergence is achieved for the objective function value. The linear programming subproblem in each iteration of the SLP is solved using the package DSPLP from the SLATEC library [23].

Uniform values of $a_{n}$ and $b_{n}$ are used as an initial guess in the static case. In the dynamic case, the solution of the static case is used as the initial guess. By using an uniform initial guess for the values of the design variables, although the solution obtained is optimized, it is not physically appropriate due to an excessive amount of grey scale (intermediate densities). This happens because the higher values of mean transduction prevents from the lower values of mean compliance.

\subsection{Example}

Two-dimensional topologies of flextensional transducers will be shown to illustrate the method. Actuator applications are considered. Figures 14(a) and 14(b) describe the design domain used to generate the results below. The domain consists of piezoceramic that remains unchanged during the optimization and a domain $S$ of brass (elastic material) where the optimization is conducted. Electrical degrees of freedom are considered only in the certain domain. The 


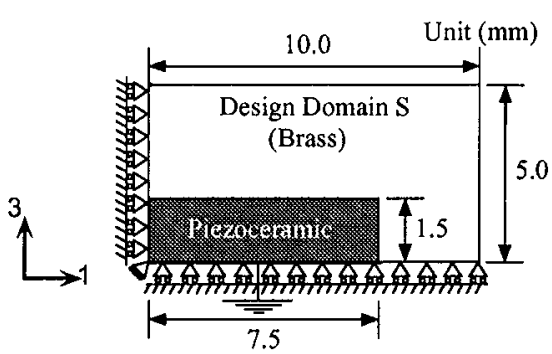

(a)

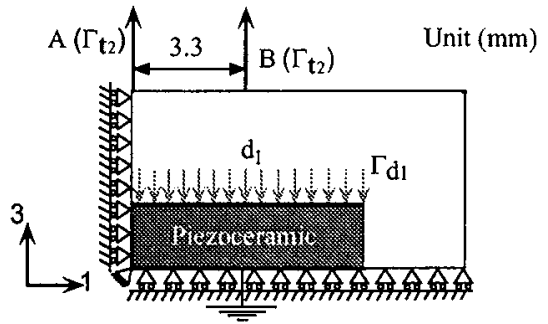

(b)

Figure 14. Design domain considered.

Table I. Material properties of PZT5.

\begin{tabular}{lccc}
\hline$c_{11}^{\mathrm{E}}\left(10^{10} \mathrm{~N} / \mathrm{m}^{2}\right)$ & 12.1 & $\mathrm{e}_{13}\left(\mathrm{C} / \mathrm{m}^{2}\right)$ & -5.4 \\
$c_{12}^{\mathrm{E}}\left(10^{10} \mathrm{~N} / \mathrm{m}^{2}\right)$ & 7.54 & $\mathrm{e}_{33}\left(\mathrm{C} / \mathrm{m}^{2}\right)$ & 15.8 \\
$c_{13}^{\mathrm{E}}\left(10^{10} \mathrm{~N} / \mathrm{m}^{2}\right)$ & 7.52 & $\mathrm{e}_{15}\left(\mathrm{C} / \mathrm{m}^{2}\right)$ & 12.3 \\
$c_{33}^{\mathrm{E}}\left(10^{10} \mathrm{~N} / \mathrm{m}^{2}\right)$ & 11.1 & $\varepsilon_{11}^{\mathrm{S}} / \varepsilon_{0}$ & 1650 \\
$c_{44}^{\mathrm{E}}\left(10^{10} \mathrm{~N} / \mathrm{m}^{2}\right)$ & 2.30 & $\varepsilon_{33}^{\mathrm{S}} / \varepsilon_{0}$ & 1700 \\
$c_{66}^{\mathrm{E}}\left(10^{10} \mathrm{~N} / \mathrm{m}^{2}\right)$ & 2.10 & $\rho\left(\mathrm{kg} / \mathrm{m}^{3}\right)$ & 5000.0 \\
\hline
\end{tabular}

domain has 800 elements $(40 \times 20$ mesh $)$. Both domains have two symmetry axes, therefore, only one-quarter of the domain is considered. The corresponding mechanical and electrical boundary conditions are described in the same figure. Two-dimensional elements under plane strain assumption are used in the finite element analysis.

The piezoelectric material properties used in the simulations are described in Table I. Young's modulus, Poisson's ratio, and density of brass are equal to $106 \mathrm{GPa}, 0.3$ and $8550.0 \mathrm{~kg} / \mathrm{m}^{3}$, respectively.

To obtain the static solution in all examples below, the total volume constraint of the material $\Theta_{s}$ was considered to be 30 per cent of the volume of the whole domain $\Omega$ without piezoceramic (domain $S$ ), and the initial values of the microscopic design variables $a_{n}$ and $b_{n}$ were 0.9 , and that of $\theta_{n}$ was 0.0 for all elements. In all cases, the amount of electrical charge applied to the piezoceramic electrode is $4 \mu \mathrm{C} / \mathrm{m}^{2}$. Any value can be applied since the analysis problem is linear.

When the optimization process is complete, the result is a material distribution over the mesh with some intermediate values of density ('grey scale') that represents the presence of perforated (composite) or intermediate material. The interpretation of the results was done through a simple threshold of the topology optimization image obtained.

4.4.1. Example 1. This example illustrates the design of a novel type of flextensional actuator. The initial design domain and load condition considered for this design are shown in Figure 14(b).

In this case, the actuator must deform along the direction specified at point $B$ in the direction specified by the dummy load when the electrical charges $d_{1}$ are applied to the 


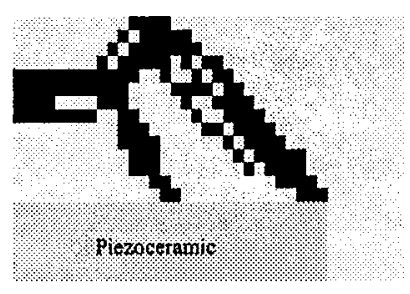

(a)

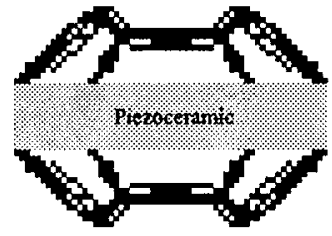

(b)

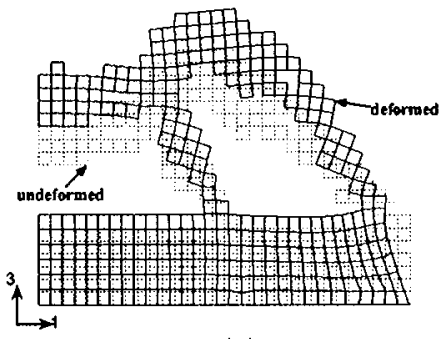

(c)

Figure 15. (a) Topology optimization result; (b) corresponding interpreted image; (c) FEM deformed image.

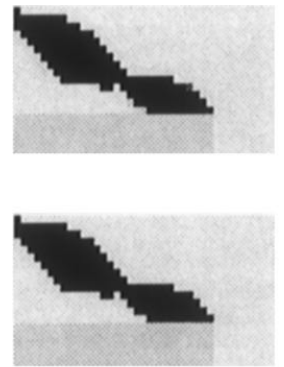

(a)
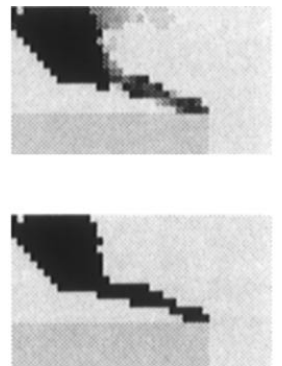

(b)
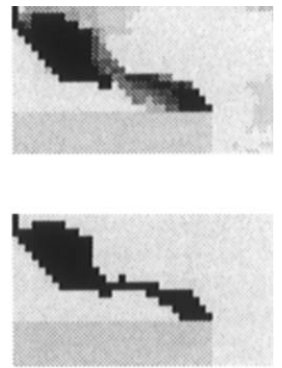

(c)

Figure 16. (a) Static solution $(w=0.7)$ and corresponding interpreted image; (b) final topology for $\omega=15 \mathrm{kHz}$ and corresponding interpreted image; (c) same for $\omega=25 \mathrm{kHz}$.

piezoceramic electrode $\Gamma_{d_{1}}$ (see Figure 14(b)). The mean transduction between regions $\Gamma_{d_{1}}$ and $B$ is maximized while the mean compliance is minimized at point $B$ where the actuator is supposed to have contact with a body. A static operation is considered, therefore $\omega=0$. The weight coefficient $w$ was considered equal to 0.5 .

The coupling structure shown in Figure 15(a) was obtained using the proposed method (the figure must be reflected to both symmetry axes). Figures 15(b) and 15(c) show, respectively, the image of the entire actuator, and the corresponding deformed configuration obtained using FEM.

4.4.2. Example 2. Figures 16(b) and 16(c) show the actuator topologies obtained by specifying excitation frequencies equal to 15 and $25 \mathrm{kHz}$, respectively, and corresponding image interpretations (the figures must be reflected to both symmetry axes). In this example, the optimization problem is defined as the maximization of the deflection at point $A$ in the direction specified by the dummy load when periodically oscillating electrical charges $d_{1}$ are applied to the piezoceramic at electrode $\Gamma_{d_{1}}$ (see Figure 14(b)) in a specified frequency $\omega$. The mean compliance at point $A$ is to be minimized since the actuator is supposed to have contact with a body at point $A$.

In both cases, the initial configuration used was the optimal configuration for the static case for the following problem: maximization of the deflection at point $A$ in the direction of 
Table II. Weighting coefficient $w$, volume constraint $\Theta_{s}$, and lowest eigenfrequency of the results shown in the figures.

\begin{tabular}{lcccc}
\hline Figure & $\omega(\mathrm{kHz})$ & $w$ & $\Theta_{S}(\%)$ & Lowest eigenfrequency $(\mathrm{kHz})$ \\
\hline $16(\mathrm{a})$ & 0 & 0.7 & 30 & 30.7 \\
$16(\mathrm{~b})$ & 15 & 0.7 & 30 & 15.1 \\
$16(\mathrm{c})$ & 25 & 0.9 & 30 & 24.9 \\
\hline
\end{tabular}

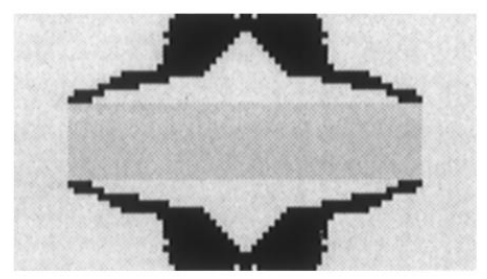

(a)

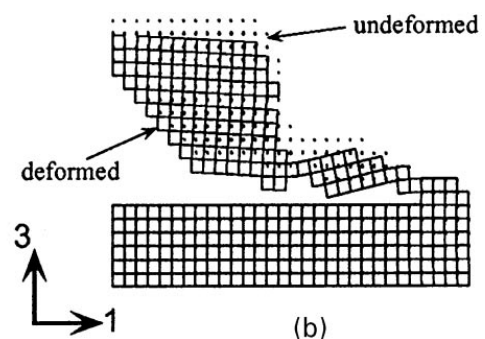

(b)

Figure 17. (a) Final actuator image; (b) corresponding first eigenmode obtained using FEM.

the dummy load when electrical charges $d_{1}$ are applied to the piezoceramic at electrode $\Gamma_{d_{1}}$ (see Figure 14(b)), while the mean compliance at point $A$ is to be minimized. The coefficient $w$ was considered equal to 0.7 and $\omega=0 \mathrm{~Hz}$. The coupling structure obtained for this static problem is shown in Figure 16(a) (the figure must be reflected to both symmetry axis).

The coefficient $w$, the total volume constraint of the material $\Theta_{s}$, the specified frequency $\omega$, and the lowest eigenfrequency obtained for the final topology are described in Table II for these examples.

Therefore, it is noted in this example that the dynamic effect affects the topology configuration, and must be considered in the design phase, especially in the high-frequency case (Figure 16).

Figures 17(a) and 17(b) show the image of the actuator obtained by reflecting Figure 16(b) to both symmetry axes, and the corresponding first eigenmode obtained using FEM considering one-quarter symmetry.

The presence of a small amount of grey scale in the final topologies suggests that some filter must be applied in a further implementation. The elimination of the grey scale due to the interpretation process will result in a small difference between the resonance frequencies of the topology result and the intepreted image. This problem could be solved by applying a parametric or shape optimization to the interpreted image.

\section{DESIGN OF ELASTIC AND THERMOELASTIC MATERIALS}

The objective of material design is to generate composite materials with prescribed or improved properties that cannot be found in the usual materials. This can be achieved by modifying the microstructure of the composite materials. The current approaches for composite 


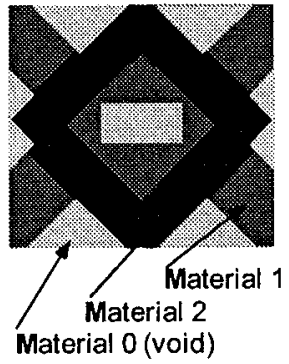

(a)

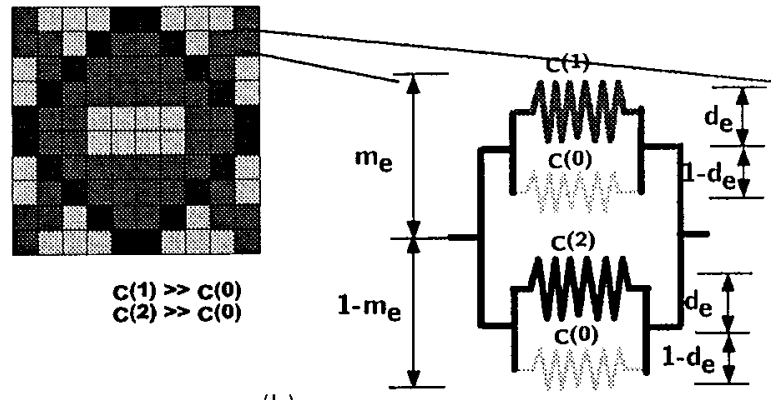

(b)

Figure 18. (a) Problem formulation: distribution of three materials in the unit cell domain: (b) discretization by FEM and schematic representation of the mixing rule at the element level.

material design have been confined to a specific subclass of configurations. The designer of laminates composites, for example, are limited by this pre-determined fibre-matrix layout $[24,25]$. Material designs are carried out by choosing among these parameters, namely, fibre ratio, lamina thickness, ply orientations and stacking sequences. In a more general nonparametric design methods, however, the designer is not bound by a pre-determined class of design layout at the onset. By using topology optimization, a systematic and wide approach for designing these materials can be provided. Therefore, a richer class of material properties can be achieved, and new kinds of composite materials can be generated rather than the usual fibre-reinforced or laminate types. The methodology based on structural topology optimization techniques to find the topology of material microstructure, in particular, has been employed to design material with specific mechanical, piezoelectric and thermal conductivity properties [26-29]. These new materials may have unusual properties such as negative Poisson's behaviour [30-32] and negative thermal expansion coefficients [27].

In this section, a method based on topology optimization combined with homogenization method for designing elastic or thermoelastic microstructures with prescribed elastic or thermoelastic properties is described. The optimization problem is formulated as to minimize this norm of the difference between the current and the specified material properties. The method is general and the only limitations for the material is imposed by the physics law which can be stated as the effective properties bounds [33,34]. A general homogenization method applied to thermoelasticity was implemented using the finite element method, to calculate the effective properties of the composite. This homogenization has no limitations regarding volume fraction or shape of the composite constituents, and is based upon assumptions of periodicity of the microstructure and the separation of the microstructure scale from the component scale through an asymptotic expansion.

\subsection{Problem formulation}

The objective is to design a microstructure with specified thermoelastic properties by distributing two or three material phases within the unit cell domain as shown in Figure 18(a). In other words, we design the topology of materials microstructures so that the designed material yields prescribed thermoelastic properties. The problem is formulated to minimize the norm of the difference between the specified and homogenized material properties. The 
homogenized material properties are obtained by conducting the homogenization analysis based on the topology of the base cell. Detailed derivations for the homogenization equations and the numerical implementation will be presented in Section 5.3. By minimizing this norm with some appropriate constraints, one should be able to find the material microstructures with thermoelastic properties close to the specified ones if the specified targets fall within physically obtainable bounds. The optimization problem can be stated as:

Minimize $F(\mathbf{x})$

the difference between the specified and homogenized properties

Variables $\mathbf{x}=\left[x_{1}, x_{2}, \ldots, x_{n}, \ldots, x_{\mathrm{NDV}}\right]$

material distribution of two or three phases within the base cell

subject to volume fraction constraints on the constituent phases bounds on the design variables lower bounds constraint on the stiffness geometry symmetry preference on the material distribution

5.1.1. Objective functions. The objective function is the $L_{2}$ norm of the difference between the specified and homogenized components of the thermoelastic tensors. Mathematically, it can be written as

$$
F=\sum_{I \in S}\left(C_{I}^{(*)}-C_{I}^{\mathrm{H}}\right)^{2}
$$

where $C_{I}^{(*)}$ and $C_{I}^{\mathrm{H}}$ are the components of the specified and homogenized thermoelastic tensors, respectively, and $S$ is the set of specified component indexes. It may be necessary to append appropriate scaling factors to the objective functions where different sets of thermoelastic properties are calculated. For example, when both components of the elasticity and thermal expansion tensors are specified such scaling is necessary since these two sets of properties are of different order.

5.1.2. Design variables and material distribution. Following the idea of structural topology optimization procedure, the topology optimization of material microstructures is transformed into the material distribution problem in which the constituent phases and the void are optimally distributed. In the discretized design domain of the base cell, the base cell is discretized by an FEM mesh. Therefore, the material distribution becomes assigning constituent phases 1 , 2 and, void to each finite element. Each element assumes constant material properties of either phase 1, 2, or void. Furthermore, this problem is relaxed by allowing the material properties of each element to vary smoothly from one constituent to another. As a result, the effective property of each element is a mixture of the three constituent phases [35].

The design variables are selected to parameterize the material local properties between the constituent phases with a simple mixture assumption. For three-phase material design, two parameters, $m_{e}$ and $d_{e}$, are selected as the design variables for element $e$ to form the mixture rule. Local elasticity tensor $\mathbf{C}^{(e)}$ and thermal stress tensor $\beta^{(e)}$ are:

$$
\begin{aligned}
\mathbf{C}^{(e)}\left(m_{e}, d_{e}\right) & =\left(d_{e}\right)^{p}\left[m_{e} \mathbf{C}^{(1)}+\left(1-m_{e}\right) \mathbf{C}^{(2)}\right] \\
\beta^{(e)}\left(m_{e}, d_{e}\right) & =\left(d_{e}\right)^{p}\left[m_{e} \beta^{(1)}+\left(1-m_{e}\right) \beta^{(2)}\right]
\end{aligned}
$$


where $\mathbf{C}^{(1)}$ and $\mathbf{C}^{(2)}$ are elastic tensors of phases 1 and $2 ; \beta^{(1)}$ and $\beta^{(2)}$ are thermal stress tensors of phases 1 and 2 . The element mixing coefficient, $m_{e} \in[0,1]$, represents the mixing ratio between the two solid phases within an element. When $m_{e}=0$ the properties assume the value of the first material, while $m_{e}=0$ recovers the properties of the second material. The element density, $d_{e} \in\left[d_{\min }, 1\right]$, denotes void element when $d_{e}=d_{\min }$, where $d_{\min }$ is a small number to avoid singularity of the stiffness matrix in the finite element calculation. Schematically, this material model corresponds to a combination of two Voigt models concatenated in parallel as shown in Figure 18(b) where phase 0 is much weaker than phases 1 and 2 to simulate the void phase.

This local material mixing assumption can be regarded as a direct extension of the classical SIMP material model which parameterizes between one solid phase and void. The power, $p$, is a penalty factor to discourage the intermediate density in the final solution. It has been shown [6] that for an isotropic material with Poisson's ration $v=\frac{1}{3}$, this penalty factor must satisfy $p \geqslant 3$ to stay within the Hashin-Shtrikman bounds [33], thus making it physically realizable.

5.1.3. Constraints on volume fraction. The volume fraction of the three constituent phases, $V^{(1)}, V^{(2)}$ and $V^{(0)}$, at the discretized domain can be found as

$$
V^{(1)}=\frac{1}{|Y|} \sum_{e=1}^{\mathrm{NE}} d_{e}\left(m_{e}\right)\left|Y^{e}\right|, \quad V^{(2)}=\frac{1}{|Y|} \sum_{e=1}^{\mathrm{NE}} d_{e}\left(1-m_{e}\right)\left|Y^{e}\right|, \quad V^{(0)}=1-V^{(1)}-V^{(2)}
$$

where $|Y|$ and $\left|Y^{e}\right|$ are the measure of the base cell and element $e$, respectively. For the problem when one wants to specify the volume fraction constraints on the constituent phases, it can be bounded by the lower $\left(V_{\min }^{(1)}, V_{\min }^{(2)}\right)$ and upper bounds $\left(V_{\max }^{(1)}, V_{\max }^{(2)}\right)$ of the component phases.

5.1.4. Constraints on stiffness. The microstructures with unusual thermal expansion properties are achieved by reducing the stiffness of the composite material in some direction. The bounds on the effective thermal expansion proposed by Gibiansky and Torquato [34] predict that materials with negative thermal expansion or very high expansion are inherently weak. Therefore, lower bound constraints on the effective stiffness are specified to avoid microstructures without significant stiffness. Written in the notation commonly used in optimization literature, the stiffness constraint can be denoted as $\left(g_{\min }\right)_{i} \leqslant g_{i}\left(\mathbf{C}^{\mathrm{H}}\right)$ for each specified stiffness. The most effective stiffness constraint is the shear modulus, $\left(C_{1212}\right)_{\min } \leqslant g\left(\mathbf{C}^{\mathrm{H}}\right)=C_{1212}^{\mathrm{H}}$, since it only involves one stiffness coefficient. For a more general case, one might want to constrain both the horizontal and vertical stiffness coefficients, namely $\left(C_{1111}\right)_{\min } \leqslant g\left(\mathbf{C}^{\mathrm{H}}\right)=C_{1111}^{\mathrm{H}}$ and $\left(C_{2222}\right)_{\min } \leqslant g\left(\mathbf{C}^{\mathrm{H}}\right)=C_{2222}^{\mathrm{H}}$.

\subsection{Optimization procedure}

The mathematical programming method of SLP was used in the numerical implementation of the optimization problem. The algorithm replaced the original optimization problem by a sequence of linear programming problems [36]. In each optimization iteration, the objective function and constraints are replaced by linear approximations obtained form the Taylor series 
expansion about current design point $\mathbf{x}^{\langle k\rangle}=\left\{\mathbf{m}^{\langle k\rangle}, \mathbf{d}^{\langle k\rangle}\right\}$. The linear programming subproblem is posed to find optimal design change $\Delta \mathbf{x}$ from the current design point:

$$
\begin{array}{ll}
\text { Minimize } & F\left(\mathbf{x}^{\langle k\rangle}\right)+\left.\sum_{n=1}^{\mathrm{NDV}}\left(\Delta x_{n}\right)\left(\frac{\partial F}{\partial x_{n}}\right)\right|_{\mathbf{x}^{\langle k\rangle}} \\
\text { Variables } & \Delta \mathbf{x}=\mathbf{x}^{\langle k+1\rangle}-\mathbf{x}^{\langle k\rangle} \\
\text { subject to } & g_{i}^{\min }-g_{i}\left(\mathbf{x}^{\langle k\rangle}\right) \leqslant\left.\sum_{n=1}^{\mathrm{NDV}}\left(\Delta x_{n}\right)\left(\frac{\partial g}{\partial x_{n}}\right)\right|_{\mathbf{x}^{\langle k\rangle}} \\
& V_{\min }^{(m)}-V^{(m)}\left(\mathbf{x}^{\langle k\rangle}\right) \leqslant\left.\sum_{n=1}^{\mathrm{NDV}}\left(\Delta x_{n}\right)\left(\frac{\partial V^{(m)}}{\partial x_{n}}\right)\right|_{\mathbf{x}^{\langle k\rangle}} \leqslant V_{\max }^{(m)}-V^{(m)}\left(\mathbf{x}^{\langle k\rangle}\right) \\
& \left(\Delta x_{n}\right)_{\min } \leqslant \Delta x_{n} \leqslant\left(\Delta x_{n}\right)_{\max }
\end{array}
$$

The last set of constraints are move limits, with $\left(\Delta x_{n}\right)_{\max }$ and $\left(\Delta x_{n}\right)_{\min }$ being the upper and lower bounds, on the allowable change in the design variable change $\Delta x_{n}$. The move limit bounds are important since the optimization iteration may never converge without a proper choice of move limits. Typically, during one iteration, the design variables will be allowed to change by $5-15$ per cent of their original values.

After optimization of the subproblem, a new set of design variables, $\mathbf{x}^{\langle k+1\rangle}=\mathbf{x}^{\langle k\rangle}+\Delta \mathbf{x}$, is obtained and updated in the unit cell. As a result, the unit cell has a new topology with effective properties closer to the specified targets. A new homogenization calculation is conducted and the convergence is verified by comparing the value of the objective function with the value in the previous iterations. The iteration proceeds until convergence in the objective function.

The linearization of the problem (Taylor series) in each iteration requires the gradients (sensitivities) of the objective function and constraints relative to the design variables. The correctness of the sensitivity analysis is of pivotal importance in solving the gradient-based optimization problems, such as SLP and optimization criteria (OC) methods. The sensitivities of the homogenized coefficient are derived in the appendix. The derivation is based on the procedure of adjoint sensitivity analysis [37, 38]. The sensitivities can be obtained from the results of the homogenization calculation and no additional adjoint problem is required for the gradient calculation.

The equations for the homogenization and its numerical solution will be presented next in details. The linear programming subproblem in each iteration of the SLP is solved using the package DSPLP from the SLATEC library [23].

The problem is inherently non-unique. For different starting points, we do get different unit cells, or at least the many definitions of periodicity for the same unit cell. Different mesh sizes may imply different resulting topologies.

Two numerical problems usually associated with the topology optimization, namely the checkerboard and mesh-dependency, are addressed by standard procedures widely adopted in the literature [39]. Checkerboards are patterns of alternating solid and void elements usually found in topology optimization results and are caused by inaccurate numerical modelling of low-order finite elements $[40,41]$. The mesh-dependency is a non-covergent phenomenon in which the greater the refinement of the finite element discretization, the more complicated the layout pattern. The filter algorithm based on image processing techniques is used to average 
out the density of each element with its immediate neighbours. By using a fixed-size filter, one can obtain a mesh-independent topology which is less prone to a checkerboard pattern even if for a refined mesh model [10].

\subsection{Homogenization formulation derivation}

The standard homogenization is a method to compute average constitutive parameters of a complex material. The homogenization method can be used for periodic materials, which are composed of a periodic repetition of a base cell. The size of the microstructure is assumed to be small compared to the size of the component. The derivation of the homogenization equation has been widely treated in literature. The reader is referred to Sanchez-Palencia [42] the reference therein for the theoretical development and Guedes and Kikuchi [43] for the numerical implementation.

Considering the standard homogenization procedure, the unit cell is defined as $Y=\left[0, Y_{1}\right] \times$ $\left[0, Y_{2}\right] \times\left[0, Y_{3}\right]$ and the material functions $c_{i j k l}$, and $\beta_{i j}$ are considered to be $Y$-periodic functions:

$$
\begin{aligned}
\mathbf{c}^{\varepsilon}(\mathbf{x}) & =\mathbf{c}(\mathbf{x}, \mathbf{y}), & \boldsymbol{\beta}^{\varepsilon}(\mathbf{x}) & =\boldsymbol{\beta}(\mathbf{x}, \mathbf{y}) \\
\mathbf{c}^{\varepsilon}(\mathbf{x}, \mathbf{y}) & =\mathbf{c}(\mathbf{x}, \mathbf{y}+Y), & \boldsymbol{\beta}^{\varepsilon}(\mathbf{x}, \mathbf{y}) & =\boldsymbol{\beta}(\mathbf{x}, \mathbf{y}+Y)
\end{aligned}
$$

and $\mathbf{y}=\mathbf{x} / \boldsymbol{\varepsilon}$ where $\boldsymbol{\varepsilon}>0$ is a small-valued parameter which represents the ratio between the micro-scale and macro-scale.

Expanding the displacement field $\mathbf{u}^{\varepsilon}$ asymptotically, we get

$$
\mathbf{u}^{\varepsilon}=\mathbf{u}_{0}(x)+\varepsilon \mathbf{u}_{1}(\mathbf{x}, \mathbf{y})
$$

where the first-order variation terms, $\mathbf{u}_{1}$, are $Y$-periodic and belongs to the space $H_{\text {per }}^{1}\left(Y, R^{3}\right)$ :

$$
\begin{aligned}
H_{\text {per }}^{1}(Y) & =\left\{v \in H^{1}(Y) \mid v \text { takes equal values on opposite sides of } Y\right\} \\
H_{\text {per }}^{1}\left(Y, R^{3}\right) & =\left\{\mathbf{v}=\left(v_{i}\right) \mid v_{i} \in H_{\text {per }}^{1}(Y), \quad i=1,2,3\right\}
\end{aligned}
$$

space. The strain field in the unit cell is written as

$$
\frac{\mathrm{d} u_{i}^{\varepsilon}}{\mathrm{d} x_{j}}=\frac{\partial u_{0 i}(\mathbf{x})}{\partial x_{j}}+\boldsymbol{\varepsilon} \frac{\partial u_{1 i}(\mathbf{x}, \mathbf{y})}{\partial x_{j}}+\frac{\partial u_{1 i}(\mathbf{x}, \mathbf{y})}{\partial y_{j}}
$$

As the parameter $\varepsilon \rightarrow 0$, the strain $\varepsilon^{\varepsilon}$ field admits the following decomposition:

$$
\varepsilon\left(\mathbf{u}^{\varepsilon}\right)=\mathbf{E}+\varepsilon_{\mathbf{y}}\left(\mathbf{u}_{1}(\mathbf{x}, \mathbf{y})\right), \quad \mathbf{E}=\varepsilon_{\mathbf{x}}\left(\mathbf{u}_{0}\right)
$$

where $\boldsymbol{\varepsilon}$ is the mechanical strain and

$$
\left(\varepsilon_{\mathbf{x}}\right)_{i j}(\cdot)=\frac{1}{2}\left(\frac{\partial(\cdot)_{i}}{\partial x_{j}}+\frac{\partial(\cdot)_{j}}{\partial x_{i}}\right) \quad\left(\varepsilon_{\mathbf{y}}\right)_{i j}(\cdot)=\frac{1}{2}\left(\frac{\partial(\cdot)_{i}}{\partial y_{j}}+\frac{\partial(\cdot)_{j}}{\partial y_{i}}\right)
$$

It is noted that the small first-order variation $\mathbf{u}_{1}$ contributes to a zero-order effect on the strain $\boldsymbol{\varepsilon}^{\varepsilon}$. Physically, this decomposition denotes that the local strain field $\boldsymbol{\varepsilon}\left(\mathbf{u}^{\varepsilon}\right)$ can be regarded as the superposition of the overall strain $\mathbf{E}$ and the local fluctuation $\boldsymbol{\varepsilon}_{\mathbf{y}}\left(\mathbf{u}_{1}\right)$. For a particular 
case when the material is homogeneous, the local fluctuation $\varepsilon_{\mathbf{y}}\left(\mathbf{u}_{1}\right)$, which accounts for the presence of heterogeneities, shall vanish and $\varepsilon\left(\mathbf{u}^{\varepsilon}\right)=\mathbf{E}$. Additionally, due to periodicity of $\mathbf{u}_{1}$, the average of the local deviation $\varepsilon_{\mathbf{y}}\left(\mathbf{u}_{1}\right)$ within the unit cell vanishes and the averages of the local strain $\varepsilon^{\varepsilon}$ equals the macroscopic strain $\mathbf{E}$ :

$$
\left\langle\boldsymbol{\varepsilon}_{\mathbf{y}}\left(\mathbf{u}_{1}\right)\right\rangle=0, \quad\left\langle\boldsymbol{\varepsilon}\left(\mathbf{u}^{\varepsilon}\right)\right\rangle=\mathbf{E}, \quad\langle f\rangle=\frac{1}{|Y|} \int_{Y} f \mathrm{~d} \mathbf{Y}
$$

where the angle bracket $\langle\cdot\rangle$ denotes the average of a physical quantity of the unit cell.

The effective material properties can be determined by solving the local problem. Under a uniform temperature rise $\Delta T$, the constitutive law for each constituent phase, the equilibrium equations, and the periodicity boundary conditions for the local problem are:

$$
\begin{aligned}
& \boldsymbol{\sigma}(\mathbf{x}, \mathbf{y})=\mathbf{c}(\mathbf{x}, \mathbf{y}):\left(\mathbf{E}+\boldsymbol{\varepsilon}_{\mathbf{y}}\left(\mathbf{u}_{1}(\mathbf{x}, \mathbf{y})\right)\right)-\boldsymbol{\beta}(\mathbf{x}, \mathbf{y}) \Delta T \quad \forall \mathbf{y} \in Y \\
& \nabla \cdot \boldsymbol{\sigma}(\mathbf{x}, \mathbf{y})=\mathbf{0}, \\
& \mathbf{u}_{1} \in H_{\mathrm{per}}^{1}\left(Y, R^{3}\right), \quad \boldsymbol{\sigma} \cdot \mathbf{n} \text { opposite on opposite sides of } \partial Y
\end{aligned}
$$

This problem admits a solution $\mathbf{u}_{1}$ which is unique up to an additive constant vector corresponding to a rigid-body translation in the periodic displacement field. On the other hand, the local strain variation $\varepsilon_{\mathbf{y}}\left(\mathbf{u}_{1}\right)$ and the stress field $\boldsymbol{\sigma}$ are uniquely defined. The variational formulation for the local problem can be seated as

$$
\left\langle\boldsymbol{\varepsilon}_{\mathbf{y}}(\mathbf{v}): \mathbf{c}: \boldsymbol{\varepsilon}_{\mathbf{y}}\left(\mathbf{u}_{1}\right)\right\rangle=-\left\langle\boldsymbol{\varepsilon}_{\mathbf{y}}(\mathbf{v}): \mathbf{c}: \mathbf{E}\right\rangle+\left\langle\boldsymbol{\varepsilon}_{\mathbf{y}}(\mathbf{v}): \boldsymbol{\beta}\right\rangle \Delta T \quad \forall \mathbf{v} \in H_{\text {per }}^{1}\left(Y, R^{3}\right)
$$

The local problem can be regarded as loaded by the overall strain $\mathbf{E}$ and the thermal loading $\boldsymbol{\beta}$. Due to the linear nature of this problem, the problem can be split into the two subproblems, loaded by the elastic strain $\mathbf{E}$ and thermal loads $\boldsymbol{\beta}$, respectively. The elastic $\mathbf{E}$ strain can be further decomposed into a linear combination of its elementary strain states:

$$
\mathbf{E}=E_{p q} \mathbf{I I}^{p q}, \quad I I_{i j}^{p q}=\frac{1}{2}\left(\delta_{i p} \delta_{j q}+\delta_{i q} \delta_{j p}\right)
$$

Therefore, the microscopic equations are obtained as

$$
\left\langle\boldsymbol{\varepsilon}_{\mathbf{y}}(\mathbf{v}): \mathbf{c}: \boldsymbol{\varepsilon}_{\mathbf{y}}\left(\chi^{p q}\right)\right\rangle=\left\langle\boldsymbol{\varepsilon}_{\mathbf{y}}(\mathbf{v}): \mathbf{c}: \mathbf{I I}^{p q}\right\rangle \quad \forall \mathbf{v} \in H_{\text {per }}^{1}\left(Y, R^{3}\right)
$$

and

$$
\left\langle\boldsymbol{\varepsilon}_{\mathbf{y}}(\mathbf{v}): \mathbf{c}: \boldsymbol{\varepsilon}_{\mathbf{y}}(\boldsymbol{\Psi})\right\rangle=\left\langle\boldsymbol{\varepsilon}_{\mathbf{y}}(\mathbf{v}): \boldsymbol{\beta}\right\rangle \quad \forall \mathbf{v} \in H_{\text {per }}^{1}\left(Y, R^{3}\right)
$$

where the characteristic displacement $\chi^{p q}$ are the solutions of the local problem when $\mathbf{E}=-\mathbf{I I}^{p q}$ and $\Delta T=0$, whereas the characteristic displacement $\boldsymbol{\Psi}$ is the result of free mechanical macroscopic strain, $\mathbf{E}=\mathbf{0}$, and the unit temperature rise, $\Delta T=1$. As a result, the overall strain becomes

$$
\boldsymbol{\varepsilon}\left(\mathbf{u}^{\varepsilon}\right)=E_{p q}\left(\mathbf{I I}^{p q}-\boldsymbol{\varepsilon}_{\mathbf{y}}\left(\chi^{p q}\right)\right)+\boldsymbol{\varepsilon}_{\mathbf{y}}(\boldsymbol{\Psi}) \Delta T
$$

Once the local problem equations (33), (34) are solved, the homogenized stiffiness $\mathbf{c}^{\mathrm{H}}$ and the homogenized thermal stress tensor $\boldsymbol{\beta}^{\mathrm{H}}$ are determined through the relationship:

$$
\mathbf{c}^{\mathrm{H}}: \mathbf{E}-\boldsymbol{\beta}^{\mathrm{H}} \Delta T=\langle\boldsymbol{\sigma}\rangle=\left\langle\mathbf{c}: \boldsymbol{\varepsilon}\left(\mathbf{u}^{\boldsymbol{\varepsilon}}\right)-\boldsymbol{\beta} \Delta T\right\rangle
$$




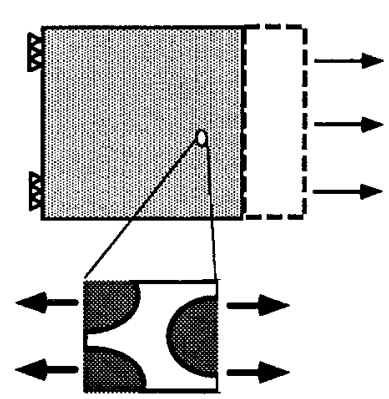

(a)

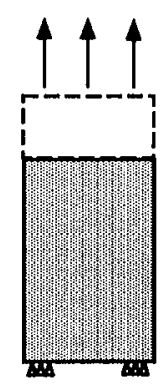

(b)

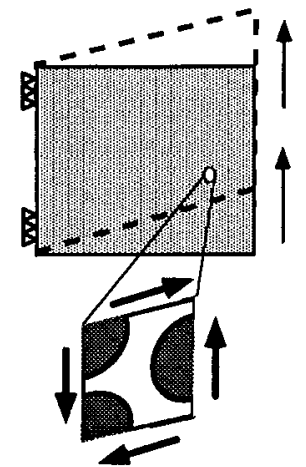

(c)

Figure 19. Three load cases: (a) subject to $I I^{11}$; (b) subject to $I I^{22}$; (c) subject to $I I^{12}$.

therefore, it yields

$$
\begin{aligned}
c_{i j p q}^{\mathrm{H}} & =\left\langle c_{i j k h}\left(I I_{k h}^{p q}-\left(\boldsymbol{\varepsilon}_{\mathbf{y}}\right)_{k h}\left(\chi^{p q}\right)\right)\right\rangle \\
\beta_{i j}^{\mathrm{H}} & =\left\langle\beta_{i j}-\left(\boldsymbol{\varepsilon}_{\mathbf{y}}\right)_{i j}(\boldsymbol{\Psi})\right\rangle
\end{aligned}
$$

Alternatively, the symmetric form for the homogenized tensors can be obtained from Equations (33), (34) and Equations (35), (36):

$$
\begin{aligned}
c_{i j k l}^{\mathrm{H}} & =\left\langle\left(\mathbf{I I}^{i j}-\varepsilon_{\mathbf{y}}\left(\boldsymbol{\chi}^{i j}\right)\right): \mathbf{c}:\left(\mathbf{I I}^{k l}-\varepsilon_{\mathbf{y}}\left(\boldsymbol{\chi}^{k l}\right)\right)\right\rangle \\
\beta_{i j}^{\mathrm{H}} & =\left\langle\left(\mathbf{I I}^{i j}-\varepsilon_{\mathbf{y}}\left(\boldsymbol{\chi}^{i j}\right)\right): \mathbf{c}:\left(\boldsymbol{\alpha}-\varepsilon_{\mathbf{y}}(\boldsymbol{\Psi})\right)\right\rangle
\end{aligned}
$$

and the effective thermal strain is

$$
\alpha_{i j}^{\mathrm{H}}=\left(c_{i j k l}^{\mathrm{H}}\right)^{-1} \beta_{k l}^{\mathrm{H}}
$$

5.3.1. Numerical implementation of homogenization. The homogenization method requires the solution of the local problem equations (33), (34) for the characteristic displacement $\chi^{p q}$ and $\boldsymbol{\Psi}$ subject to unit independent macroscopic strain II $^{p q}$ and unit temperature rise $\Delta T=1$.

In two-dimensional space, for example, the microscopic characteristic displacements $\chi^{11}, \chi^{22}$ and $\chi^{12}$ are the solution fields of the corresponding macroscopic unit strain $\mathbf{I I}^{11}, \mathbf{I I}^{22}$ and $\mathbf{I I}^{12}$, namely two tensile strains and one shear strain, as shown in Figure 19.

The solution of the local problem equations (33), (34) are solved by applying the finite element method. The unit cell is discretized by NE finite elements, that is

$$
Y=\bigcup_{n=1}^{\mathrm{NE}} \Omega^{e}
$$

where $\Omega^{e}$ is the domain of each element. Hexagonal elements with eight-node brick elements were used for the $3 \mathrm{D}$ case, and four-node incompatible elements were used for the $2 \mathrm{D}$ case. 
Trilinear and bilinear interpolation functions were considered for displacements in the 3D and 2D case, respectively. Therefore, the characteristic functions previously defined are expressed in each element as a function of the shape functions $\left(N_{I}\right)$ :

$$
\chi_{i}^{p q} \cong N_{I} \chi_{i I}^{p q}, \quad \psi_{i} \cong N_{I} \psi_{i I}, \quad I=1, \mathrm{NN}
$$

where $\mathrm{NN}$ is the number of nodes per finite element. A similar relation hold for the virtual displacement $\mathbf{v}$. Replacing the approximation in Equation (33) yields [43]

$$
\sum_{e=1}^{\mathrm{NE}} \int_{\Omega_{e}} c_{i j k h} \frac{\partial N_{I}}{\partial y_{h}} \chi_{k I}^{p q} \frac{\partial N_{J}}{\partial y_{j}} v_{i J} \mathrm{~d} \Omega^{e}=\sum_{e=1}^{\mathrm{NE}} \int_{\Omega_{e}} c_{i j p q} \frac{\partial N_{J}}{\partial y_{j}} v_{i J} \mathrm{~d} \Omega^{e}
$$

and finally the following matrix equations for each independent load case $p q$

$$
\mathbf{K} \chi^{p q}=\mathbf{f}^{p q}
$$

where the global stiffness matrix is the assembly of all the element individual matrices and the global force vectors are the assembly of all the element individual force vectors

$$
\mathbf{K}=\sum_{e=1}^{\mathrm{NE}} \mathbf{K}^{e} \quad \mathbf{f}^{p q}=\sum_{e=1}^{\mathrm{NE}} \mathbf{f}^{e(p q)}
$$

The element matrices and force vectors are expressed as [43]

$$
K_{i I j J}^{e}=\int_{\Omega_{e}} c_{i k j h} \frac{\partial N_{I}}{\partial y_{h}} \frac{\partial N_{J}}{\partial y_{k}} \mathrm{~d} \Omega^{e}, \quad f_{i I}^{e(p q)}=\int_{\Omega_{e}} c_{i j p q} \frac{\partial N_{I}}{\partial y_{j}} \mathrm{~d} \Omega^{e}
$$

When calculating the thermal expansion characteristic function $\psi$, we solve an additional problem

$$
\mathbf{K} \psi=\mathbf{f}^{\text {th }}
$$

where $\mathbf{K}$ is the same as in the elastic case, and the global force vector for the thermal expansion case is an assemblage of the elements individual vectors

$$
\mathbf{f}^{\text {th }}=\sum_{e=1}^{\mathrm{NE}} \mathbf{f}^{e(\mathrm{th})}, \quad f_{i I}^{e(\mathrm{th})}=\int_{\Omega_{e}} \beta_{i j} \frac{\partial N_{I}}{\partial y_{j}} \mathrm{~d} \Omega^{e}
$$

After solving for the characteristic displacements, the effective properties can be obtained using Equations (35) and (36). Assuming that the base cell is discretized by NE finite elements, the effective properties can be calculated through the expression [44]:

$$
c_{i j p q}^{\mathrm{H}}=\frac{1}{|Y|} \sum_{e=1}^{\mathrm{NE}} \int_{\Omega^{e}} c_{i j k h}\left(I I_{k h}^{p q}-\left(\boldsymbol{\varepsilon}_{y}\right)_{k h}\left(\chi^{p q}\right)\right) \mathrm{d} \Omega^{e}
$$

or employing the symmetric form the homogenized coefficient using Equations (37) and (38) as a sum of element mutual energies $Q_{I}^{e}[27]$ :

$$
c_{i j p q}^{\mathrm{H}}=\sum_{e=1}^{\mathrm{NE}} Q_{I}^{e}
$$


where

$$
Q_{I}^{e}=\frac{1}{\left|Y^{e}\right|} \int_{\Omega^{e}}\left(I I_{r s}^{i j}-\left(\boldsymbol{\varepsilon}_{y}\right)_{r s}\left(\chi^{i j}\right)\right): c_{r s p q}:\left(I I_{p q}^{k l}-\left(\boldsymbol{\varepsilon}_{y}\right)_{p q}\left(\chi^{k l}\right)\right) \mathrm{d} \Omega^{e}
$$

and $\left|Y^{e}\right|$ is the measure of element $e ; I$ is the compact one-dimensional index equivalent to the four-free indices $i j k l$, ranging over the $N I$ independent elastic coefficients $(6$ for $2 \mathrm{D}$ or 21 for 3D). Similarly, we can define the thermal stress coefficients as a sum of element mutual energies:

$$
\beta_{k l}^{\mathrm{H}}=\sum_{e=1}^{\mathrm{NE}} B_{I}^{e}
$$

where

$$
B_{I}^{e}=\frac{1}{\left|Y^{e}\right|} \int_{\Omega^{e}}\left(I I_{p q}^{i j}-\left(\boldsymbol{\varepsilon}_{y}\right)_{p q}\left(\chi^{i j}\right)\right) c_{p q r s}\left(\alpha_{r s}-\left(\boldsymbol{\varepsilon}_{y}\right)_{r s}(\Psi)\right) \mathrm{d} \Omega^{e}, \quad e=1, \ldots, \mathrm{NE}, I=1, \ldots, \mathrm{NTH}
$$

where $I$ now spans the NTH independent thermal expansion coefficients ( 3 for $2 \mathrm{D}$ and 6 for $3 \mathrm{D})$.

The four-node bilinear and four-node Taylor non-conforming elements [45] were implemented for 2D problems, and the eight-node trilinear and the eight-node Taylor non-conforming elements [45] were implemented for 3D problems [44].

\subsection{Numerical example of material microstructure design}

5.4.1. Negative Poisson's ratio material. In this example, a two-phase (solid and void) microstructure is designed with a specified negative Poisson's ratio. The component phases are isotropic. The first phase of the base material has Young's modulus $C^{(1)}=0.91(\mathrm{GPa})$ and Poisson's ratio $v^{(1)}=0.3$ whereas the second phase is much weaker, $C^{(2)}=0.91 \times 10^{-4}(\mathrm{GPa})$ and $v^{(2)}=0.3$, to simulate the void phase. Under the plane stress assumption, the components of the elastic tensor of the base material are $C_{1111}^{(1)}=C_{2222}^{(1)}=1.0, C_{1122}^{(1)}=0.3$ and $C_{1212}^{(1)}=0.35$. It should not be a serious concern for designing with this kind of 'normalized' base material since for the linear elastic material with the same Poisson's ratio, the effective material properties obtained for the real material simply differ by a scale factor.

The specified material properties are, $C_{1111}^{*}=C_{2222}^{*}=0.4(\mathrm{GPa}), C_{1122}^{(*)} / C_{1111}^{(*)}=-0.5$ under the volume ratio constraint $V^{(1)}=30$ per cent. The vertical symmetry of the density distribution is enforced. The optimization problem formulation can be stated as

$$
\begin{aligned}
& \min \left[C_{1111}^{\mathrm{H}}-C_{1111}^{*}\right]^{2}+\left[C_{2222}^{\mathrm{H}}-C_{2222}^{*}\right]^{2}+\left[C_{1122}^{\mathrm{H}}-C_{1122}^{*}\right]^{2} \\
& \mathbf{X}=\left[d_{1}, d, \ldots, d_{\mathrm{NE}}\right] \\
& \text { subject to } 0.3 \leqslant V^{(1)} \leqslant 0.3 \\
& \qquad 0.0<d_{\min } \leqslant d_{e} \leqslant 1.0, \quad e=1, \ldots, \mathrm{NE} \\
& \text { vertical symmetry }
\end{aligned}
$$




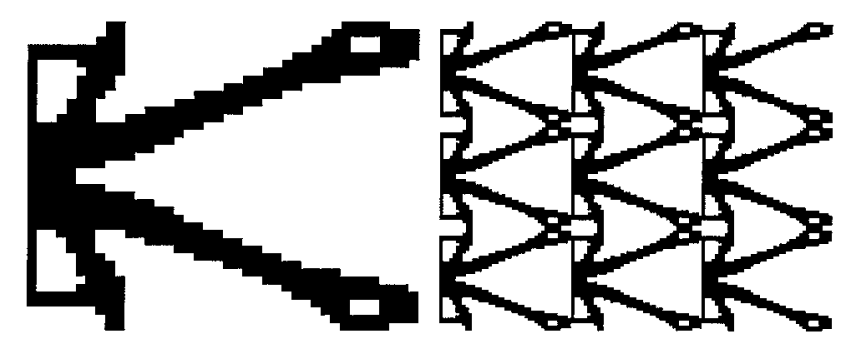

Figure 20. Negative Poisson's ratio material microstructures with vertical density symmetry.

The base cell is discretized by a $40 \times 40$ mesh. The layout of the microstructure unit cell and the composite materials composed of a $3 \times 3$ array of the base cells are shown in Figure 20 . The black region denotes the solid phase and the white region is void. It is noted this layout is actually an inverted structure and is very similar to the result obtained by Larsen et al. [27]. The final achieved properties are found to be

$$
C^{\mathrm{H}}=0.04\left[\begin{array}{ccc}
1.0 & -0.496 & 0 \\
-0.496 & 0.995 & 0 \\
0 & 0 & 0.315
\end{array}\right]
$$

5.4.2. Negative thermal stress material. In next two examples, we will demonstrate the design of a three-phase microstructure with 'normalized' materials. The first and second phases have the same rigidity and Poisson's ratio, $C^{(1)}=C^{(2)}=0.91(\mathrm{GPa}), v^{(1)}=v^{(2)}=0.3$ but different thermal expansion coefficients, $\alpha^{(1)}=1.0\left(\mu \mathrm{m} /{ }^{\circ} \mathrm{C}\right)$ and $\alpha^{(2)}=10.0\left(\mu \mathrm{m} /{ }^{\circ} \mathrm{C}\right)$. The void phase is simulated with a weak material which is insignificant in its rigidity compared with the two bulk phases. The objective is to construct microstructures with the specified thermal stress tensor, $\beta_{11}^{*}=\beta_{22}^{*}=-1.0\left(\mathrm{kPa} /{ }^{\circ} \mathrm{C}\right)$, under the volume fraction constraints $0.20 \leqslant V^{(1)} \leqslant 0.25$ and $0.20 \leqslant V^{(2)} \leqslant 0.25$. The lower bound constraint on the shear stiffness is imposed to ensure sufficient rigidity of the resulting microstructure. The problem formulation can be stated as

$$
\begin{aligned}
& \min \left[\beta_{11}^{\mathrm{H}}-\beta_{11}^{*}\right]^{2}+\left[\beta_{22}^{\mathrm{H}}-\beta_{22}^{*}\right]^{2} \\
& \mathbf{X}=\left[d_{1}, m_{1}, d_{2}, m_{2}, \ldots d_{\mathrm{NE}}, m_{\mathrm{NE}}\right] \\
& \text { subject to } \\
& \begin{array}{l}
0.20 \leqslant V^{(1)} \leqslant 0.25, \quad 0.20 \leqslant V^{(2)} \leqslant 0.25 \\
0.05 \leqslant C_{1212}^{\mathrm{H}} \\
0.0<d_{\min } \leqslant d_{e} \leqslant 1.0, \quad 0.0 \leqslant m_{e} \leqslant 1.0
\end{array}
\end{aligned}
$$

vertical symmetry

The model is discretized by a $30 \times 30$ mesh. The resulting microstructure is shown in Figure 21 with the achieved properties, $\beta_{11}^{*}=-1.003$ and $\beta_{22}^{*}=-1.031\left(\mathrm{kPa} /{ }^{\circ} \mathrm{C}\right)$. 

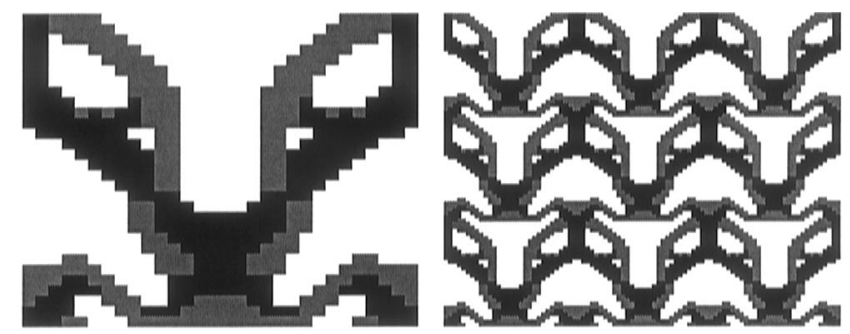

Figure 21. Negative thermal stress material microstructure with vertical density symmetry.
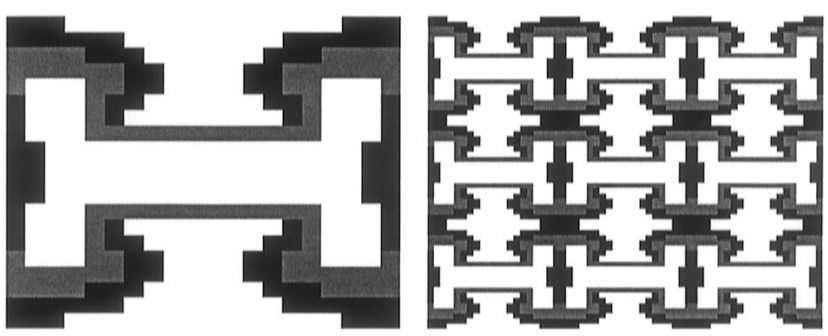

Figure 22. Horizontal high expansion microstructures with horizontal and vertical symmetries.

5.4.3. High thermal expansion material. In this example, we will demonstrate the design of a three-phase microstructure with high thermal expansion properties in the horizontal direction. The target material property is $\alpha_{11}^{*}=20.0\left(\mu \mathrm{m} /{ }^{\circ} \mathrm{C}\right)$. Under the volume fraction constraints $0.15 \leqslant V^{(1)} \leqslant 0.20$ and $0.15 \leqslant V^{(2)} \leqslant 0.20$. The lower bound constraints on the stiffness, $0.05 \leqslant C_{1111}^{\mathrm{H}}$ and $0.05 \leqslant C_{2222}^{\mathrm{H}}$, are imposed. Both horizontal and vertical symmetries on the distribution pattern are specified.

The final layout of the unit cell of the microstructure is shown in Figure 22 with the achieved properties, $\alpha_{11}^{\mathrm{H}}=19.6\left(\mu \mathrm{m} /{ }^{\circ} \mathrm{C}\right)$. The fact that the composite material expands more than the constituent phases results from the deformation pattern of the bi-material composite arch structure as can be seen from the figure. The higher expansion phase is located inside this curved structure. When heated, the inside strip expands more than the outside low expansion strip and causes the overall structure to bend outward thus resulting in high expansion in the horizontal direction.

5.4.4. Negative thermal strain microstructure composed of real materials. In this example, we will design a negative thermal expansion material microstructure composed of real materials. Chromium is selected as the first phase and Nickel as the second phase with properties $C^{(1)}=290(\mathrm{GPa}), v^{(1)}=0.3, \alpha^{(1)}=4.9(\mu \mathrm{m} / \mathrm{K})$ and $C^{(2)}=200(\mathrm{GPa}), v^{(2)}=0.3$, $\alpha^{(2)}=13.4(\mu \mathrm{m} / \mathrm{K})$. It should be noted that chromium and nickel are admissible to each other so one can expect to have good bonding at the bi-material interface. The objective is to construct a microstructure with the specified thermal strain components, $\alpha_{11}^{*}=\alpha_{22}^{*}=-4.0(\mu \mathrm{m} / \mathrm{K})$, under the volume fraction constraints, $0.20 \leqslant V^{(1)} \leqslant 0.25, \quad 0.20 \leqslant V^{(2)} \leqslant 0.25$, and the lower bound constraint on the shear stiffness. 


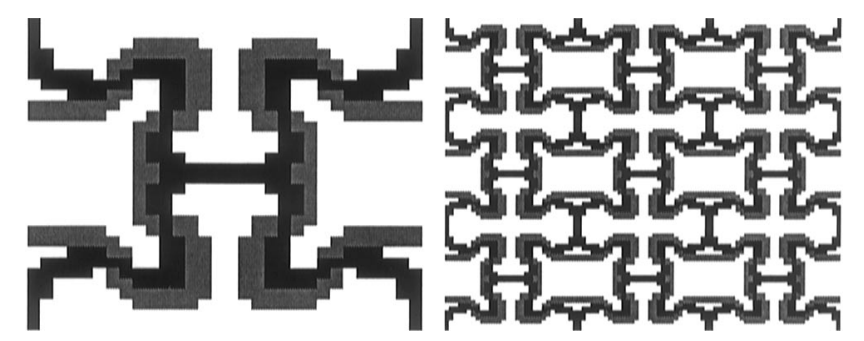

Figure 23. Negative thermal strain material microstructure with horizontal and vertical symmetries. The black colour denotes chromium and gray color is nickel.

The problem formulation can be stated as

$$
\begin{aligned}
& \min \left[\alpha_{11}^{\mathrm{H}}-\alpha_{11}^{*}\right]^{2}+\left[\alpha_{22}^{\mathrm{H}}-\alpha_{22}^{*}\right]^{2} \\
& \mathbf{X}=\left[d_{1}, m_{1}, d_{2}, m_{2}, \ldots d_{\mathrm{NE}}, m_{\mathrm{NE}}\right] \\
& \text { subject to } \\
& 0.20 \leqslant V^{(1)} \leqslant 0.25, \quad 0.20 \leqslant V^{(2)} \leqslant 0.25 \\
& 0.05 \leqslant C_{1212}^{\mathrm{H}} \\
& 0.0<d_{\min } \leqslant d_{e} \leqslant 1.0, \quad 0.0 \leqslant m_{e} \leqslant 1.0 \\
& \text { vertical and horizontal symmetry }
\end{aligned}
$$

This model was initially discretized by a $30 \times 30$ mesh with a uniform density distribution. A rudimentary layout was obtained with $\alpha_{11}^{\mathrm{H}}=-3.2, \alpha_{22}^{*}=-4.8(\mu \mathrm{m} / \mathrm{K})$. Then each element was further divided into four elements with identical material properties to form a refined model with a $60 \times 60$ mesh. The optimization process is then continued with this refined model. The final layout of the material microstructure is shown in Figure 23. The resulting properties are closer to the specified target as

$$
\alpha^{\mathrm{H}}=\left[\begin{array}{cc}
-3.993 & 0 \\
0 & -4.027
\end{array}\right](\mu \mathrm{m} / \mathrm{K})
$$

Using the strategy of starting with a rough model and using a rudimentary layout as the starting topology in the refined model, a considerable amount of computing time is saved. As a result, one can adjust the discretization refinement depending on the accuracy demand and the available computing resources. It is interesting to note this microstructure is very similar to the result obtained by Torquato and Sigmund [27] while the base cell is shifted by half of the cell in the horizontal direction.

In order to visualize the shrinkage effect of the designed microstructure, a verification simulation for the structure made of an array of $3 \times 3$ microstructures was conducted using the commercial package MSC/NASTRAN. The structure is subject to a unit temperature rise under the free expansion condition. The deformation of the repeated structure is shown in Figure 24. As can be seen from the figure, the bi-material deformation effect of chromium and nickel contributes to the overall shrinkage of the material. This microstructure is made of several connecting bi-material arches. Each arch is composed of two curved strips in 


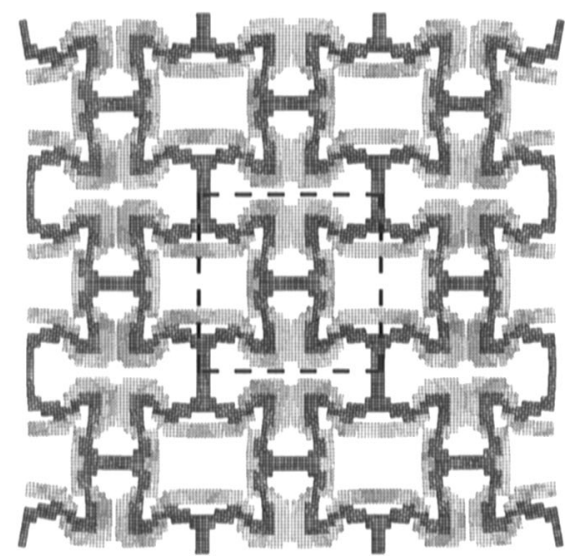

Figure 24. Deformation of $3 \times 3$ array of negative thermal expansion microstructures.

which the higher expansion phase is located outside and lower expansion phase is situated inside. Contrary to the bi-material arch structure for high expansion material in Figure 22, this layout arrangement causes the cell to bend inward and thus contract. It is noted that this curved bi-material arch has been used as the basic building block to construct temperatureinsensitivity structures [46] with application in space structures where structural distortion caused by temperature fluctuation is a major concern.

5.4.4.1. Manufacturing and experiments. The design for the negative thermal expansion microstructures were fabricated using direct metal deposition (DMD) techniques [47]. DMD is a layer-based additive manufacturing process that uses a laser to melt powdered metals and make deposits. The laser is used in conjunction with a CNC workstation. The object is fabricated on top of a substrate material, which is attached to the CNC motion table. The motion of the table provides the relative motion between the substrate and the laser beam, allowing the geometry of the deposit tracks to be controlled through CNC code. The $\mathrm{CNC}$ code for the negative thermal expansion microstructure is generated from a CAD/CAM system which interprets the density distribution of the topology optimization result into a CAD model. This interpretation is accomplished by the following steps: thresholding the densities, removing isolated noise elements, and interpolating curves.

DMD has the capability of making intricate structures that would be otherwise impossible to make using conventional manufacturing techniques, especially for multi-material designs. Since it is an additive technique, changing composition is simply changing the composition of the powder being sent to the laser work area, thus making DMD a good candidate for the meso-scale manufacturing process for the designed microstructures.

The manufactured structure is tested to verify the results. The specimen is placed within a three-zone furnace. The designed structure was tested in the horizontal direction at a heating rate of $3.5(\mathrm{~K} / \mathrm{min})$. The measured thermal expansion coefficient, $\alpha_{22}=-3.9(\mu \mathrm{m} / \mathrm{K})$, is in good agreement with the predicted design target (Figure 25). 


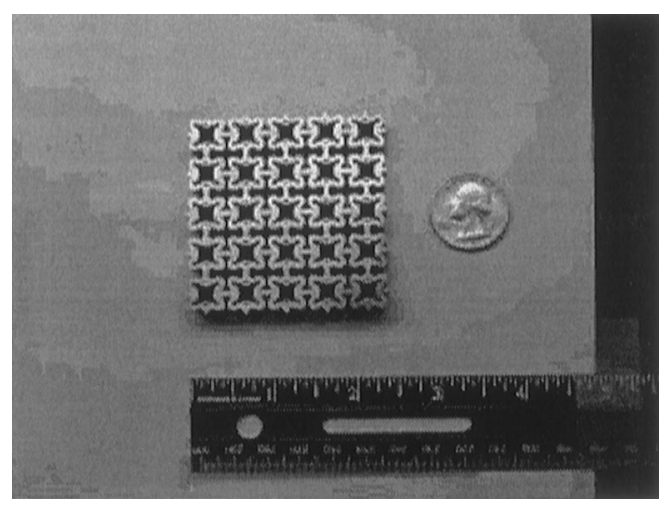

Figure 25. An array of $5 \times 5$ negative thermal expansion microstructures built with direct metal deposition techniques. (Courtesy of Mr Eric Stiles and Dr Jyotirmoy Mazumder of the Center for Laser Aided Intelligent Manufacturing at the University of Michigan.)

\section{CONCLUSIONS}

The application of topology optimization for designing pressure actuated compliant mechanisms, flextensional transducers, and porous material microstructures with unusual thermoelastic properties has been presented. By changing the layout of the sensors/actuators, novel types of transducers for different tasks can be designed. The examples in this paper span different physics domain. The flextensional transducer spans the electric and elastic fields via piezoceramics. The compliant mechanism actuated by hydrostatic pressure serves as a starting point to design more intricate device involving solid and fluid interaction. The material microstructure with unusual thermal expansion coefficients can be regarded as a thermal actuator in a miniature scale. These examples demonstrate that the topology optimization problem involving multiple physics domain is a viable direction for future research, in particular, for sensor and actuator design where various performance goals are imposed for different applications.

\section{APPENDIX A: SENSITIVITY ANALYSIS OF THE HOMOGENIZED COEFFICIENTS}

The sensitivities used in the microstructure design optimization are derived in this appendix. This derivation is based on the adjoint sensitivity analysis applied on the homogenization coefficients, Equations (35), (36), which are determined from the characteristic displacement fields obtained from the homogenization equations (33), (34). The derivation procedure starts with a non-symmetric form of the homogenization coefficients formula and ends up with a symmetric form for the gradient of the homogenization coefficients.

\section{A.1. Sensitivity of the homogenized elastic tensor}

Without loss of generality, we will begin with deriving the sensitivity of the first component of the homogenized elastic tensor, $c_{1111}^{\mathrm{H}}$. This component of the homogenized elastic coefficient, Equation (35), written in the notation commonly adopted in the structural optimization 
literature:

$$
g=\int_{Y} g\left(\mathbf{b}, \varepsilon\left(\chi^{11}\right)\right) \mathrm{d} Y=c_{1111}^{\mathrm{H}}=\left\langle c_{11 k h}\left(I I_{k h}^{11}-\varepsilon_{k h}\left(\chi^{11}\right)\right)\right\rangle
$$

where the function $g$ is continuously differentiable with respect to its arguments, namely, the design variable $\mathbf{b}$ and the 'state variables' of the characteristic equations $\chi^{11} \in H_{\text {per }}^{1}\left(Y, R^{3}\right)$ and $\varepsilon\left(\chi^{11}\right)$, which are determined by the 'state equation', Equation (33), written in the indicial notation:

$$
\left\langle\varepsilon_{i j}(\mathbf{v}) c_{i j k h} \varepsilon_{k h}\left(\chi^{11}\right)\right\rangle=\left\langle\varepsilon_{i j}(\mathbf{v}) c_{i j 11}\right\rangle \quad \forall \mathbf{v} \in H_{\text {per }}^{1}\left(Y, R^{3}\right)
$$

Take first variation of $G$

$$
\begin{aligned}
\delta G & =\int_{Y}\left[\frac{\partial g}{\partial \mathbf{b}} \delta \mathbf{b}+\frac{\partial g}{\partial \boldsymbol{\varepsilon}} \delta \boldsymbol{\varepsilon}\right] \mathrm{d} Y \\
& =\left\langle\delta c_{11 k h}\left(I I_{k h}^{11}-\varepsilon_{k h}\left(\chi^{11}\right)\right)\right\rangle+\left\langle c_{11 k h} \frac{\partial\left(I I_{k h}^{11}-\varepsilon_{k h}\left(\chi^{11}\right)\right)}{\partial \varepsilon_{p q}\left(\chi^{11}\right)} \delta \varepsilon_{p q}\left(\chi^{11}\right)\right\rangle \\
& =\left\langle\delta c_{11 k h}\left(I I_{k h}^{11}-\varepsilon_{k h}\left(\chi^{11}\right)\right)\right\rangle-\left\langle c_{11 p q} \delta \varepsilon_{p q}\left(\chi^{11}\right)\right\rangle
\end{aligned}
$$

The explicit term, $(\partial g / \partial \mathbf{b}) \delta \mathbf{b}$, depends on the change in the direction of design $\delta \mathbf{b}$ directly. On the other hand, in the implicit part $(\partial g / \delta \boldsymbol{\varepsilon}) \delta \boldsymbol{\varepsilon}$, the dependency on design change $\delta \mathbf{b}$ needs to go through the variation of the strain field $\delta \varepsilon\left(\chi^{11}\right)$. It is noted since the periodicity boundary conditions are not dependent on the design change, both the characteristic displacement field $\chi^{11}$ and its variation $\delta \chi^{11}$ belong to the kinematic admissible space, namely, $\chi^{11} \in H_{\text {per }}^{1}$ and $\delta \chi^{11} \in H_{\text {per }}^{1}$.

In order to evaluate the implicit part in terms of the design change $\delta \mathbf{b}$ explicitly without going through the dependency relationship of $\delta \boldsymbol{\varepsilon}$, the procedure of adjoint sensitivity analysis is employed $[37,38]$. The adjoint equation corresponding to the pseudo-load term, $\partial g / \partial \boldsymbol{\varepsilon}$, is

$$
\left\langle\varepsilon_{i j}(\mathbf{z}) c_{i j k h} \varepsilon_{k h}\left(\lambda^{11}\right)\right\rangle=\left\langle\varepsilon_{i j}(\mathbf{z})\left(c_{11 i j}\right)\right\rangle \quad \forall \mathbf{z} \in H_{\text {per }}^{1}\left(Y, R^{3}\right)
$$

Taking the first variation of the state equation, Equation (A2), one gets an equation involving $\delta \chi^{11}$ :

$$
\left\langle\varepsilon_{i j}(\mathbf{v}) c_{i j k h} \varepsilon_{k h}\left(\delta \chi^{11}\right)\right\rangle=\left\langle\varepsilon_{i j}(\mathbf{v}) \delta c_{i j 11}\right\rangle-\left\langle\varepsilon_{i j}(\mathbf{v}) \delta c_{i j k h} \varepsilon_{k h}\left(\chi^{11}\right)\right\rangle \quad \forall \mathbf{v} \in H_{\text {per }}^{1}\left(Y, R^{3}\right)
$$

Substitute $\delta \chi^{11}$ for $\mathbf{z}$ in Equation (A4) and substitute $\lambda^{11}$ for $\mathbf{v}$ in Equation (A5) and using the symmetry property, $c_{i j k h}=c_{k h i j}$, the implicit term becomes

$$
\begin{aligned}
\left\langle\varepsilon_{i j}\left(\delta \chi^{11}\right)\left(c_{11 i j}\right)\right\rangle & =\left\langle\varepsilon_{i j}\left(\delta \chi^{11}\right) c_{i j k h} \varepsilon_{k h}\left(\lambda^{11}\right)\right\rangle=\left\langle\varepsilon_{i j}\left(\delta \lambda^{11}\right) c_{i j k h} \varepsilon_{k h}\left(\delta \chi^{11}\right)\right\rangle \\
& =\left\langle\varepsilon_{i j}\left(\lambda^{11}\right) \delta c_{i j k h}\left(I I_{k h}^{11}-\varepsilon_{k h}\left(\chi^{11}\right)\right)\right\rangle
\end{aligned}
$$

Up to this point, the implicit term can be represented explicitly by the design change $\delta c_{i j k h}$. Substitute this term back into Equation (A3)

$$
\delta c_{1111}^{\mathrm{H}}=\left\langle\left(I I_{i j}^{11}-\varepsilon_{i j}\left(\lambda^{11}\right)\right) \delta c_{i j k h}\left(I I_{k h}^{11}-\varepsilon_{k h}\left(\chi^{11}\right)\right)\right\rangle
$$


Furthermore, it is noted that the adjoint equation (A4) and the state equation (A2) are identical. In other words, one may identify the solution of the adjoint equation, $\lambda^{11}$, with the solution of the state equation, $\chi^{11}$, and get a symmetry form for the sensitivity:

$$
\delta c_{1111}^{\mathrm{H}}=\left\langle\left(I I_{i j}^{11}-\varepsilon_{i j}\left(\chi^{11}\right)\right) \delta c_{i j k h}\left(I I_{k h}^{11}-\varepsilon_{k h}\left(\chi^{11}\right)\right)\right\rangle
$$

One can generalize this result for each component of the homogenized elastic tensor,

$$
\delta c_{p q r s}^{\mathrm{H}}=\left\langle\left(I I_{i j}^{r s}-\varepsilon_{i j}\left(\chi^{r s}\right)\right) \delta c_{i j k h}\left(I I_{k h}^{p q}-\varepsilon_{k h}\left(\chi^{p q}\right)\right)\right\rangle
$$

\section{A.2. Sensitivity of the thermal stress tensor}

Following the similar derivation procedure, the sensitivity analysis of the first component of the homogenized thermal stress tensor, $\beta_{11}^{\mathrm{H}}$, starts from Equation (36):

$$
G=\int_{Y} g(\mathbf{b}, \boldsymbol{\varepsilon}(\boldsymbol{\Psi})) \mathrm{d} Y=\beta_{11}^{\mathrm{H}}=\left\langle\beta_{11}-c_{11 k h} \varepsilon_{k h}(\boldsymbol{\Psi})\right\rangle
$$

and its variation:

$$
\delta \beta_{11}^{\mathrm{H}}=\left\langle\delta \beta_{11}-\delta c_{11 k h} \varepsilon_{k h}(\boldsymbol{\Psi})-c_{11 p q} \varepsilon_{p q}(\delta \boldsymbol{\Psi})\right\rangle
$$

The corresponding adjoint equation is exactly identical to Equation (A4).

The state equation (34) and its first variation, in inidicial notation:

$$
\begin{aligned}
\left\langle\varepsilon_{i j}(\mathbf{v}): c_{i j k l}: \varepsilon_{k l}(\boldsymbol{\Psi})\right\rangle & =\left\langle\varepsilon_{i j}(\mathbf{v}): \beta_{i j}\right\rangle \quad \forall \mathbf{v} \in H_{\mathrm{per}}^{1}\left(Y, R^{3}\right) \\
\left\langle\varepsilon_{i j}(\mathbf{v}) c_{i j k h} \varepsilon_{k h}(\delta \boldsymbol{\Psi})\right\rangle & =\left\langle\varepsilon_{i j}(\mathbf{v}) \delta \beta_{i j}\right\rangle-\left\langle\varepsilon_{i j}(\mathbf{v}) \delta c_{i j k h} \varepsilon_{k h}(\boldsymbol{\Psi})\right\rangle \quad \forall \mathbf{v} \in H_{\mathrm{per}}^{1}\left(Y, R^{3}\right)
\end{aligned}
$$

Substitute $\delta \boldsymbol{\Psi}$ for $\mathbf{z}$ in Equation (A4) and substitute $\lambda^{11}$ for $\mathbf{v}$ in Equation (A8) the implicit term becomes

$$
\left\langle\varepsilon_{i j}(\delta \boldsymbol{\Psi}) c_{11 i j}\right\rangle=\left\langle\varepsilon_{i j}\left(\lambda^{11}\right) \delta \beta_{i j}\right\rangle-\left\langle\varepsilon_{i j}\left(\lambda^{11}\right) \delta c_{i j k h} \varepsilon_{k h}(\boldsymbol{\Psi})\right\rangle
$$

Substitute this term back into Equation (A7)

$$
\begin{aligned}
\delta \beta_{11}^{\mathrm{H}} & =\left\langle\left(I I_{i j}^{11}-\varepsilon_{i j}\left(\lambda^{11}\right)\right) \delta \beta_{i j}\right\rangle-\left\langle\left(I I_{i j}^{11}-\varepsilon_{i j}\left(\lambda^{11}\right)\right) \delta c_{i j k h} \varepsilon_{k h}(\boldsymbol{\Psi})\right\rangle \\
& =\left\langle\left(I I_{i j}^{11}-\varepsilon_{i j}\left(\lambda^{11}\right)\right) \delta c_{i j k h}\left(\alpha_{k h}-\varepsilon_{k h}(\boldsymbol{\Psi})\right)\right\rangle+\left\langle\left(I I_{i j}^{11}-\varepsilon_{i j}\left(\lambda^{11}\right)\right) c_{i j k h} \delta \alpha_{k h}\right\rangle
\end{aligned}
$$

This formula can be further simplified by $\varepsilon_{i j}\left(\lambda^{11}\right)=\varepsilon_{i j}\left(\chi^{11}\right)$,

$$
\delta \beta_{11}^{\mathrm{H}}=\left\langle\left(I I_{i j}^{11}-\varepsilon_{i j}\left(\chi^{11}\right)\right) \delta c_{i j k h}\left(\alpha_{k h}-\varepsilon_{k h}(\boldsymbol{\Psi})\right)\right\rangle+\left\langle\left(I I_{i j}^{11}-\varepsilon_{i j}\left(\chi^{11}\right)\right) c_{i j k h} \delta \alpha_{k h}\right\rangle
$$

and the general form for each component of the homogenized thermal stress tensor,

$$
\delta \beta_{p q}^{\mathrm{H}}=\left\langle\left(I I_{i j}^{p q}-\varepsilon_{i j}\left(\chi^{p q}\right)\right) \delta c_{i j k h}\left(\alpha_{k h}-\varepsilon_{k h}(\boldsymbol{\Psi})\right)\right\rangle+\left\langle\left(I I_{i j}^{p q}-\varepsilon_{i j}\left(\chi^{p q}\right)\right) c_{i j k h} \delta \alpha_{k h}\right\rangle
$$




\section{A.3. Sensitivity of the thermal strain tensor}

The sensitivity of the homogenized thermal strain tensor $\alpha^{\mathrm{H}}$ can be found by taking variation of the relation, $\boldsymbol{\alpha}^{\mathrm{H}}=\left(\mathbf{c}^{\mathrm{H}}\right)^{-1}: \boldsymbol{\beta}^{\mathrm{H}}$, to get

$$
\begin{aligned}
\delta \boldsymbol{\alpha}^{\mathrm{H}} & =\left(\delta \mathbf{c}^{\mathrm{H}}\right)^{-1}: \boldsymbol{\beta}^{\mathrm{H}}+\left(\mathbf{c}^{\mathrm{H}}\right)^{-1}: \delta \boldsymbol{\beta}^{\mathrm{H}} \\
& =-\left(\mathbf{c}^{\mathrm{H}}\right)^{-1}:\left(\delta \mathbf{c}^{\mathrm{H}}\right): \boldsymbol{\alpha}^{\mathrm{H}}+\left(\mathbf{c}^{\mathrm{H}}\right)^{-1}: \delta \boldsymbol{\beta}^{\mathrm{H}}
\end{aligned}
$$

\section{ACKNOWLEDGEMENTS}

We are grateful to Mr Eric Stiles and Dr Jyotirmoy Mazumder for manufacturing the Negative CTE microstructures and Mr Ramkumar Oruganti and Dr Amit Ghosh for measuring the material properties. The financial support of DARPA for this research under Grant No. DARPA/NIST F000765, and the support of Sekisui Chemical Industry, is gratefully acknowledged.

\section{REFERENCES}

1. Back-Pedersen A. Taking advantage of using both topology and shape optimization for practical design. NAFEMS Word Congress 1999, Newport, Rhode Island, 1999; 889-899.

2. Bloembaum $\mathrm{C}$. The use of structural optimization in automotive design - state of the art and vision. WCSMO-3 Buffalo 1999; 200-202.

3. Kohn RV, Strang G. Optimal design in elasticity and plasticity. Numerical Methods in Engineering 1986; 22:183-188.

4. Kohn RV, Strang G. Optimal design and relaxation of variational problems. Communications on Pure and Applied Mathematics (New York) 1986; 39:1-25 (part I), 139-182 (part II) and 353-357 (part III).

5. Bendsøe MP, Kikuchi N. Generating optimal topologies in structural design using a homogenization method. Computer Methods in Applied Mechanics and Engineering 1988; 71:197-224.

6. Bendsøe MP, Sigmund O. Material interpolation schemes in topology optimization. Archive of Applied Mechanics 1999; 69:635-654.

7. Her I, Midha A. Compliance number concept for compliant mechanisms and type synthesis. Journal of Mechanisms, Transmissions, and Automotion in Design, Transactions of the ASME 1987; 109(3):348-355.

8. Nishiwaki S, Frecker MI, Min S, Kikuchi N. Topology optimization of compliant mechanisms using the homogenization method. International Journal for Numerical Methods in Engineering 1998; 42(3):535-559.

9. Frecker MI, Ananthasuresh GK, Nishiwaki S, Kikuchi N, Kota S. Topological synthesis of compliant mechanisms using multi-criteria optimization. Journal of Mechanical Design, Transactions of the ASME 1997; 19(2):238-245.

10. Sigmund O. On the design of compliant mechanisms using topology optimization. Mechanics of Structures and Machines 1997; 25(4):493-524.

11. Bendsøe MP. Optimization of Structural Topology, Shape, and Material. Springer: Berlin, 1995.

12. Bendsøe MP, Sokołowski J. Shape sensitivity analysis of optimal compliance functionals. Mechanics of Structures and Machines 1995; 23(1):35-58.

13. Hammer VB, Olhoff N. Topology optimization with design dependent loads. WCSMO-3 Buffalo, 1999; 629-631

14. Chen B-C, Kikuchi N. Topology optimization with design-dependent loads. Archive Finite Elements in Analysis and Design, in press.

15. Rolt KD. History of the flextensional electroacoustic transducer. The Journal of the Acoustical Society of America 1990; 87(3):1340-1349.

16. Dogan A, Yoshikawa S, Uchino K, Newnham RE. The effect of geometry on the characteristics of the moonie transducer and reliability issue. IEEE 1994 Ultrasonic Symposium Proceedings, 1994; 935-939.

17. Xu QC, Yoshikawa S, Belsick JR, Newnham RE. Piezoelectric composites with high sensitivity and high capacitance for use at high pressures. IEEE Transactions on Ultrasonics, Ferroelectrics and Frequency Control 1991; 38(6):634-639.

18. Dogan A, Uchino K, Newnham RE. Composite piezoelectric transducer with truncated conical endcaps 'Cymbal'. IEEE Transactions on Ultrasonics, Ferroelectrics and Frequency Control 1997; 44(3):597-605. 
19. Inoue T, Nada T, Sugiuchi K. Low-frequency flextensional piezoelectric underwater transmitter with displacement amplifier. IEEE Transactions of the IEICE 1989; E72(12):1410-1416.

20. Lerch R. Simulation of piezoelectric devices by two- and three-dimensional finite elements. IEEE Transactions on Ultrasonics, Ferroelectrics and Frequency Control 1990; 37(2):233-247.

21. Silva ECN. Design of piezocomposite materials and piezoelectric transducers using topology optimization. Ph.D. Dissertation, The University of Michigan-Ann Arbor, 1998.

22. Pedersen P. On optimal orientation of orthotropic materials. Structural Optimization 1989; 1:101-106.

23. Hanson R, Hiebert K. A sparse linear programming subprogram. Sandia National Laboratories Technical Report SAND81-0297, 1981.

24. Autio M. Coupled thermal-structure problems in the optimization of laminated plates. Structural Optimization 1998; 15:49-56.

25. Wetherhold RC, Wang J. Tailoring thermal deformation by using layered beams. Composite Science and Technology 1995; 53:1-6.

26. Sigmund O. Materials with prescribed constitutive parameters: an inverse homogenization problem. International Journal of Solids and Structures 1994; 31(17):2313-2329.

27. Sigmund O, Torquato S. Design of materials with extreme thermal expansion using a three-phase topology optimization method. Journal of the Mechanics and Physics of Solids 1997; 45(6):1037-1067.

28. Silva EC, Fonseca JSO, Kikuchi N. Optimal design of periodic piezocomposites. Computer Methods in Applied Mechanics and Engineering 1998; 159(1-2):49-77.

29. Haslinger J, Dvorak J. Optimum composite material design. RAIRO Modelisation Mathematique et Analyse Numerique 1995; 29:657-686.

30. Lakes R. Design considerations for materials with negative Poisson's ratio. Journal of Mechanical Design 1993; 115:696-700.

31. Lakes R. Foam structures with negative Poisson's ratio. Science 1987; 235:1038-1040.

32. Milton GW. Composite materials with Poisson's ratio close to -1. Journal of the Mechanics and Physics of Solids 1992; 40(5):1105-1137.

33. Hashin Z, Shtrikman S. A variational approach to the theory of the elastic behavior of multiphase materials. Journal of the Mechanics and Physics of Solids 1963; 11:127-140.

34. Gibiansky LV, Torquato S. Thermal expansion of isotropic multiphase composite and polycrystals. Journal of the Mechanics and Physics of Solids 1997; 45:1223-1252.

35. Bendsøe MP. Optimal shape design as a material distribution problem. Structural Optimization 1989; 1: 192-202.

36. Vanderplaatz GN. Numerical Optimization Techniques for Engineering Design: with Applications. McGrawHill: New York, 1984.

37. Choi KK, Haug EJ, Komkov V. Design Sensitivity Analysis of Structural Systems. Academic Press Inc.: London, 1986.

38. Dems K, Mróz Z. Variational approach by means of adjoint systems to structural optimization and sensitivity analysis-I. International Journal of Solids and Structures 1983; 19(8):677-692.

39. Petersson J, Sigmund O. Slope constrained topology optimization. International Journal for Numerical Method in Engineering 1998; 41(8):1417-1434.

40. Diaz A, Sigmund O. Checkerboard patterns in layout optimization. Structural Optimization 1995; 10:40-45.

41. Jog CS, Haber RB. Stability of finite element models for distributed-parameter optimization and topology design. Computer Methods in Applied Mechanics and Engineering 1996; 130(3-4):203-226.

42. Sanchez-Palencia E. Non-Homogeneous Media and Vibration Theory. Springer: Berlin, 1980.

43. Guedes JM, Kikuchi N. Preprocessing and postprocessing for materials based on the homogenization method with adaptive finite element methods. Computer Methods in Applied Mechanics and Engineering 1990; 83:143-198.

44. Fonseca JSO. Design of microstructures of periodic composite material. Ph.D. Dissertation, The University of Michigan, 1997.

45. Taylor RL, Beresford PJ, Wilson EL. A nonconforming element for stress analysis. International Journal for Numerical Methods in Engineering 1976; 10(6):1211-1219.

46. Ryvkin M, Sleypan L, Fuchs MB. Temperature insensitive curved bimetal elements. Acta Astronautica 1995; 37:95-103.

47. Mazumder J et al. Direct metal deposition of h13 tool steel for 3-d components. Journal of Materials 1997; 49(5):55-60.

48. Sigmund O. Tailoring materials with prescribed elastic properties. Mechanics of Materials 1995; 20:351-368. 